\title{
Highly confined mixtures of parallel hard squares: A density-functional-theory study
}

\author{
Yuri Martínez-Ratón (1) \\ Grupo Interdisciplinar de Sistemas Complejos, Departamento de Matemáticas, Escuela Politécnica Superior, Universidad Carlos III de \\ Madrid, Avenida de la Universidad 30, 28911 Leganés, Madrid, Spain \\ Enrique Velasco ${ }^{\dagger}$ \\ Departamento de Física Teórica de la Materia Condensada, Instituto de Física de la Materia Condensada and Instituto de Ciencia de \\ Materiales Nicolás Cabrera, Universidad Autónoma de Madrid, 28049, Madrid, Spain
}

(Received 16 October 2019; published 19 December 2019)

\begin{abstract}
Using the fundamental-measure density-functional theory, we study theoretically the phase behavior of extremely confined mixtures of parallel hard squares in slit geometry. The pore width is chosen such that configurations consisting of two consecutive big squares, or three small squares, in the transverse direction, perpendicular to the walls, are forbidden. We analyze two different mixtures with edge lengths of species selected so as to allow or forbid one big plus one small square to fit into the channel. For the first mixture we obtain first-order transitions between symmetric and asymmetric packings of particles: Small and big squares are preferentially adsorbed at different walls. Asymmetric configurations are shown to lead to more efficient packing at finite pressures. We argue that the stability region of the asymmetric phase in the pressure-composition plane is bounded so that the symmetric phase is stable at low and very high pressure. For the second mixture, we observe strong demixing between phases which are rich in different species. Demixing occurs in the lateral direction, i.e., the dividing interface is perpendicular to the walls, and phases exhibit symmetric density profiles. The possible experimental realization of this behavior (which in practical terms is precluded by jamming) in strictly twodimensional systems is discussed. Finally, the phase behavior of a mixture with periodic boundary conditions is analyzed and the differences and similarities between the latter and the confined system are discussed. We claim that, although exact calculations exclude the existence of true phase transitions in $(1+\epsilon)$-dimensional systems, density-functional theory is still successful in describing packing properties of large clusters of particles.
\end{abstract}

DOI: 10.1103/PhysRevE.100.062604

\section{INTRODUCTION}

Fluids of two-dimensional hard anisotropic particles are paradigmatic examples of systems exhibiting entropy-driven phase transitions to orientationally and positionally ordered phases. The elucidation of the phase behavior of twodimensional (2D) fluids composed of hard particles is not an academic study, since hard particles enjoy many experimental realizations. To cite a recent representative experiment, extreme confinement of three-dimensional lithographically synthesized prisms with different polygonal cross sections in quasi-2D geometries has been accomplished [1-4]. The phase behavior of these effectively two-dimensional Brownian particles was reported and their tendency to produce chiral phases $[1,3]$ or racemic mixtures of monomers and dimers was emphasized [2]. Also, the phase behavior of colloidal monolayers of particles with exotic shapes was reported [4]. Research on three-dimensional colloidal particles of different shapes, especially in connection with packing and partial or complete crystallization, has also been very active (see Ref. [5] for a review).

Several theoretical works have concentrated on the elucidation of the phase behavior of hard polygonal particles [6-14].

\footnotetext{
*yuri@math.uc3m.es

†enrique.velasco@uam.es
}

The results show that it strongly depends on the symmetries of particle shapes. Apart from the usual uniaxial nematic phase present in fluids of elongated rods, other more "exotic" orientational fluid phases, such as triatic, tetratic, and hexactic phases, also exist. For example, hard rectangles may order into uniaxial nematic phases, but also in tetratic arrangements at low particle aspect ratios [7,8]. Different plastic or orientationally ordered crystals have been classified as a function of particle shape $[12,13]$. Especially interesting is the case of hard squares because of their plane-filling properties and the mathematical simplicity of their interaction potential. Classical work on the numerical calculation of virial coefficients $[15,16]$ demonstrates the importance of hard squares as a simple model to elucidate important problems in statistical mechanics. The lattice-gas version of the model has attracted some attention [17-19]. The parallel hard-square model has also been investigated [20-22]. Simulations have shown that freely oriented hard squares present nematic tetratic and crystal square phases [6]. Rounded hard squares have been investigated and their phase behaviors are seen to depend on the degree of roundness [9]. An experimental realization of this system has been reported, together with evidence for a rich phase diagram [23]. Also, demixing transitions in mixtures of hard squares have been explored by simulation [24].

The effect of confinement on two-dimensional fluids of rodlike particles in cavities of square, rectangular, or circular 
geometries has been extensively studied [25-32]. When the confining geometry is incompatible with the symmetry of the bulk phase, the system usually responds to the geometric frustration by creating point defects or domain walls in the orientational field. Hard particles exhibit preferred orientations at the boundary of the confining walls, which are controlled solely by entropy. These "anchoring" effects are strong enough that the creation of defects is unavoidable. The number and symmetry of the defects strongly depends on the geometry of the confining cavity and on the symmetries of the bulk phases.

When confinement of 2D hard particles (and also of 3D hard spheres inside a cylindrical pore) between two hard lines is so extreme that the system is close to the $1 \mathrm{D}$ limit the partition function can be calculated for nearest-neighbor or next-nearest-neighbor interactions using the transfer-matrix method (TMM). This method becomes a useful (and potentially exact) theoretical tool to extract information about the structure of the confined fluid. In essence, the technique calculates, apart from the partition function, the probability density and pair correlations between particles. The method was successfully applied to the study of hard disks, squares, rhombuses, and rectangles under strong confinement [33-40]. The results can be summarized as follows. (i) Phase transitions between different spatial structures are ruled out, a confirmation of the general result that fluids composed of particles interacting via hard-core potentials do not exhibit phase transitions in $1+\epsilon$ dimensions. (ii) From the behavior of probability densities and pair correlation functions, smooth crossovers between different spatial structures can be shown to exist. For example, the system may change from a one-layer structure that behaves approximately as a 1D Tonk gas to a structure consisting of two highly correlated layers adsorbed at each wall. Correlation may be different depending on the specific particle geometry (circular vs square). (iii) Although phase transitions can be ruled out, the equation of state (EOS) may exhibit, in a range of packing fraction associated with the structural crossover, a plateau and consequently the specific heat exhibits a sharp peak in this range of packing fraction.

The implementation of the TMM for such systems serves as an ideal test bed to study the performance of available density functionals (DFs) developed for 2D fluids of hard disks [41], parallel hard squares (PHSs) [42], rectangles within the restricted orientation approximation [43], or freely rotating discorectangles [44]. All of these DFs are based on the fundamental-measure theory (FMT), initially developed for hard spheres and further extended to anisotropic particles. (For reviews of this theoretical tool see Refs. [45,46].) Recent work on highly confined PHS and rectangles (in the orientation-restricted or Zwanzig approximation) in slit geometry using both theories, the TMM and FMT, demonstrated the high performance of the FMT to predict changes in the structural properties of the fluid induced by confinement and also to describe the anomalous behavior of the EOS at the crossover between different structures $[39,47]$.

In the present article we go beyond the one-component fluid studied previously and focus on the effect of extreme confinement on the structural and thermodynamical properties of binary mixtures of PHSs, using a FMT-based formalism. Mixtures of small (edge length equal to $\sigma_{1}$ ) and big (edge length equal to $\sigma_{2}$ ) squares are confined in a channel of width $H$. The value of $H$ is selected in such a way that at most two layers of small squares can fit into the channel, whereas only one layer (but not two) of big squares can fit. We analyze two different mixtures characterized by the ratios $\sigma_{2} / \sigma_{1}=$ 1.5 and 2. We find micro- and macrosegregation first-order transitions for the first and second mixtures, respectively. In the former case, different species are preferentially adsorbed at different walls, while in the latter species phase separate, with a dividing surface perpendicular to the walls. We explain, using entropic arguments, why these mixtures segregate. We claim that a TMM applied to these mixtures could confirm the appearance of large clusters of micro- or macrosegregated particles as the packing fraction is increased, despite the fact that an exact theory should exclude the existence of a true phase transition between different structures.

The paper is organized as follows. In Sec. II the model is introduced and details are provided on the theory. Also, the numerical procedure used to find the phase behavior of the system is discussed. Technical details to prove the nonexistence of fluid-fluid demixing at the bulk phase, along with the method to find the spinodal instability of uniform phases with respect to $1 \mathrm{D}$ spatial density modulations, are relegated to Appendixes A and B, respectively. In Sec. III the results are presented. This section is divided into two parts, where results obtained for mixtures with $\sigma_{2} / \sigma_{1}=1.5$ (Sec. III A) and $\sigma_{2} / \sigma_{1}=2$ (Sec. III B) are given. The end of Sec. III B is devoted to describing the phase behavior of the $\sigma_{2} / \sigma_{1}=2$ mixture that results from imposing periodic boundary conditions, instead of a confining external potential. A summary is given and some conclusions are drawn in Sec. IV.

\section{MODEL AND THEORY}

Our model consists of a binary mixture of PHSs confined in a channel (or a slit pore) formed by two parallel hard lines (or walls) with a relative distance between them of $H$ (the pore width). See Fig. 1 for a sketch of the system. Small and large particles have edge lengths equal to $\sigma_{1}$ and $\sigma_{2}$, respectively. Coordinates parallel and perpendicular to the walls are chosen as $x$ and $y$, respectively, and the walls are located at $y=0$ and $y=H$. Our system is described in terms of the density profile of species $i, \rho_{i}(y)$, which is assumed to depend only on the $y$ coordinate. The mean density, averaged in the channel, of the $i$ th species is defined as

$$
\rho_{i} \equiv \frac{1}{H} \int_{0}^{H} d y \rho_{i}(y), \quad \rho=\rho_{1}+\rho_{2},
$$

(a)

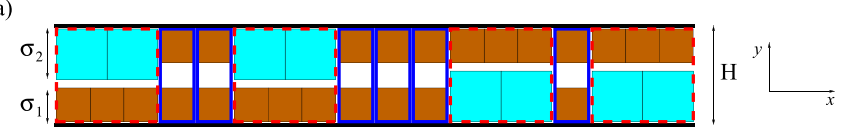

(b)

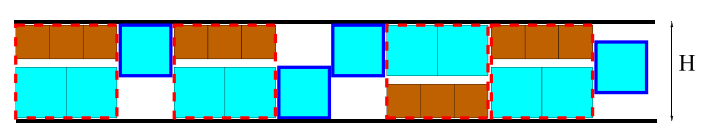

FIG. 1. Schematic of close-packing configurations of binary mixtures of PHSs with $\sigma_{2} / \sigma_{1}=1.5$ and molar fractions (a) $\mathrm{x}>3 / 5$, and (b) $x<3 / 5$. The small and big clusters are indicated with solid and dashed lines, respectively. 
with $\rho$ the total mean density. The mixture composition is described in terms of the mean molar fraction of small species:

$$
\mathbf{x} \equiv \mathbf{x}_{1}=\frac{\rho_{1}}{\rho}, \quad \mathbf{x}_{2}=\frac{\rho_{2}}{\rho}=1-\mathbf{x}, \quad \sum_{i} \mathbf{x}_{i}=1 .
$$

The mean packing fraction of the mixture is, as usual, calculated as

$$
\eta=\sum_{i=1}^{2} \eta_{i}=\sum_{i=1}^{2} \rho_{i} \sigma_{i}^{2} .
$$

The theoretical model used is a version of density-functional theory (DFT), the so-called FMT, which was formulated for PHSs in [42] and has been extensively tested before in several studies $[39,47]$. The main assumption of the theory, adapted to the present system, is that the excess (or interaction) part of the free-energy density of the PHS fluid only depends on four weighted densities

$$
\begin{gathered}
n_{0}(y)=\frac{1}{2} \sum_{i}\left[\rho_{i}\left(y_{i}^{-}\right)+\rho_{i}\left(y_{i}^{+}\right)\right], \\
n_{2}(y)=\sum_{i} \sigma_{i} \int_{y_{i}^{-}}^{y_{i}^{+}} d y^{\prime} \rho_{i}\left(y^{\prime}\right), \\
n_{1 x}(y)=\frac{1}{2} \sum_{i} \sigma_{i}\left[\rho_{i}\left(y_{i}^{-}\right)+\rho_{i}\left(y_{i}^{+}\right)\right], \\
n_{1 y}(y)=\sum_{i} \int_{y_{i}^{-}}^{y_{i}^{+}} d y^{\prime} \rho_{i}\left(y^{\prime}\right),
\end{gathered}
$$

where $y_{i}^{ \pm}=y \pm \sigma_{i} / 2$. The explicit expression for the excess free-energy density, in reduced thermal units, is [42]

$$
\Phi_{\text {exc }}(y)=-n_{0}(y) \ln \left[1-n_{2}(y)\right]+\frac{n_{1 x}(y) n_{1 y}(y)}{1-n_{2}(y)},
$$

while the ideal part, neglecting the thermal areas, is

$$
\Phi_{\mathrm{id}}(y)=\sum_{i} \rho_{i}(y)\left[\ln \rho_{i}(y)-1\right] .
$$

The grand potential per unit length can then be calculated as

$$
\frac{\Omega\left[\left\{\rho_{i}\right\}\right]}{L}=\frac{\mathcal{F}\left[\left\{\rho_{i}\right\}\right]}{L}-\sum_{i} \int_{0}^{H} d y\left[\mu_{i}-v_{\mathrm{ext}}^{(i)}(y)\right] \rho_{i}(y)
$$

with $\mathcal{F}\left[\left\{\rho_{i}\right\}\right]$ the Helmholtz free-energy DF

$$
\begin{aligned}
\frac{\beta \mathcal{F}\left[\left\{\rho_{i}\right\}\right]}{L} & =\frac{\beta \mathcal{F}_{\mathrm{id}}\left[\left\{\rho_{i}\right\}\right]}{L}+\frac{\beta \mathcal{F}_{\mathrm{exc}}\left[\left\{\rho_{i}\right\}\right]}{L} \\
& =\int_{0}^{H} d y \Phi_{\mathrm{id}}(y)+\int_{0}^{H} d y \Phi_{\mathrm{exc}}(y),
\end{aligned}
$$

with $\beta=\left(k_{B} T\right)^{-1}$ the inverse of temperature, $\mu_{i}$ the chemical potential of species $i$, and $L$ the length of the system. The external potential acting on particle $i$ is defined as

$$
\beta v_{\mathrm{ext}}^{(i)}(y)= \begin{cases}0 & \text { for } \frac{\sigma_{i}}{2} \leqslant y \leqslant H-\frac{\sigma_{i}}{2} \\ \infty & \text { otherwise }\end{cases}
$$

By minimizing the grand potential with respect to $\rho_{i}(y)$, i.e., $\frac{\delta \beta \Omega\left[\left\{\rho_{i}\right\}\right]}{\delta \rho_{i}(y)}=0$, we obtain

$$
\rho_{i}(y)= \begin{cases}e^{-\Psi_{i}(y)+\beta \mu_{i}} & \text { for } \frac{\sigma_{i}}{2} \leqslant y \leqslant H-\frac{\sigma_{i}}{2} \\ 0 & \text { otherwise }\end{cases}
$$

where we have used the shorthand notation

$$
\Psi_{i}(y) \equiv \frac{\delta \beta \mathcal{F}_{\text {exc }}\left[\left\{\rho_{i}\right\}\right] / L}{\delta \rho_{i}(y)} .
$$

The longitudinal pressure inside the channel can be calculated as

$$
\begin{aligned}
\beta p & =\frac{1}{H}\left\{\sum_{i}\left[\int_{0}^{H} d y \rho_{i}(y)\left[1+\Psi_{i}(y)\right]\right]-\frac{\beta \mathcal{F}_{\text {exc }}}{L}\right\} \\
& =\frac{1}{H} \int_{0}^{H} d y\left[\frac{n_{0}(y)}{1-n_{2}(y)}+\frac{n_{1 x}(y) n_{1 y}(y)}{\left[1-n_{2}(y)\right]^{2}}\right] .
\end{aligned}
$$

By fixing the values of both mean packing fractions $\eta_{i}$ inside the channel, the constrained minimization of the free energy $\beta \mathcal{F}\left[\left\{\rho_{i}\right\}\right]$ with respect to $\rho_{i}(y)$ leads to

$$
\rho_{i}(y)=\frac{\eta_{i} e^{-\Psi_{i}(y)}}{\sigma_{i}^{2} H^{-1} \int_{0}^{H} d y^{\prime} e^{-\Psi_{i}\left(y^{\prime}\right)}}
$$

for $\sigma_{i} / 2 \leqslant y \leqslant H-\sigma_{i} / 2$ and zero otherwise. Obviously the two routes, (i) to fix the chemical potentials $\mu_{i}$ and (ii) to fix the packing fractions $\eta_{i}$, are equivalent. Using the second route to calculate the equilibrium density profiles, the chemical potentials can be calculated as

$$
\beta \mu_{i}=\ln \left[\frac{\eta_{i}}{\sigma_{i}^{2} H^{-1} \int_{0}^{H} d y e^{-\Psi_{i}(y)}}\right] .
$$

To study the thermodynamics of the confined fluid mixture, which is necessary to calculate possible phase transitions, it is more convenient to use the Gibbs free energy per particle in reduced thermal units, defined as

$$
g \equiv \frac{\beta}{\rho}\left(\frac{\mathcal{F}}{L H}+p_{0}\right) .
$$

Here the pressure of the confined mixture is fixed,

$$
p(\mathbf{x}, \rho)=p_{0}
$$

and $\rho$ can be numerically calculated as a function of the mixture composition $\mathbf{x}$ once the equilibrium density profiles $\left\{\rho_{i}^{\text {(eq) }}(y)\right\}$ are obtained from Eq. (16). The function $g(\mathbf{x})$ can then be obtained. In the case of first-order phase transitions a double-tangent construction on $g(\mathbf{x})$ allows us to calculate the coexisting values of molar and packing fractions. For convenience, we will use a dimensionless pressure $p_{0}^{*} \equiv \beta p_{0} \sigma_{1}^{2}$.

Appendix A presents a proof that the uniform mixture of PHSs is always stable at the bulk phase, i.e., no demixing is possible. In Appendix B the spinodal instability of uniform phases with respect to one-dimensional periodic inhomogeneities is discussed by means of a bifurcation analysis.

\section{RESULTS}

This section is devoted to presenting the results obtained from the numerical solutions of Eqs. (16) and (19), which provide the equilibrium density profiles $\rho_{i}(y)$ for fixed pressure $p_{0}^{*}$ and for a given mixture composition $\mathbf{x}$. Varying $\mathbf{x}$ inside a given set of values $\left\{\mathrm{x}_{i}=i / N_{\mathrm{x}}, i=0, \ldots, N_{\mathrm{x}}, N_{\mathrm{x}} \sim 100\right\}$ allows us to obtain a sufficiently accurate Gibbs free energy per particle $g(\mathbf{x})$ [from Eq. (18)] to search for possible phase transitions and calculate the phase diagrams. This section 


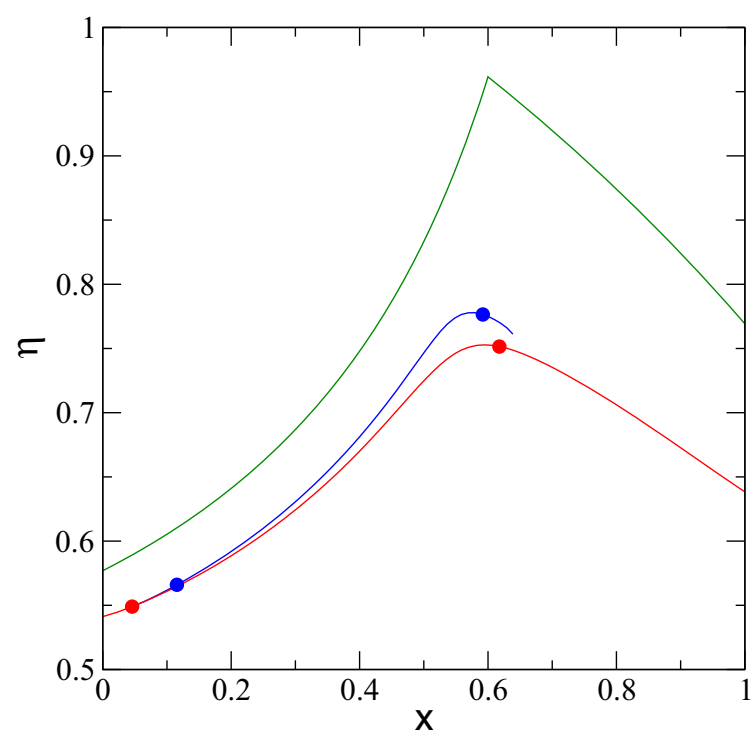

FIG. 2. Packing fraction $\eta$ vs molar fraction $\mathrm{x}$ for symmetric (red curve) and asymmetric (blue curve) configurations of a confined binary mixture of PHSs with $\sigma_{2} / \sigma_{1}=1.5, H / \sigma_{1}=2.6$, and $p_{0}^{*}=4$. The green curve corresponds to the packing fraction for the closepacked configuration $\eta_{\mathrm{CP}}$ (see the text). Note that the maxima of the curves $\eta(\mathbf{x})$ in both symmetric and asymmetric configurations are located close to $x=0.6$, the maximum close-packing value. Red and blue circles indicate the symmetric and asymmetric coexisting states, respectively. Thus the red curve between red circles corresponds to metastable states. These states also occur on the blue curve to the left and right of the blue circles.

is divided into two parts. Section III A is concerned with a confined binary mixture of PHSs with $\sigma_{2} / \sigma_{1}=1.5$ and several values of pore width $H / \sigma_{1}$. Values of $H$ were chosen to ensure that only two small squares (but not three) or one big plus one small square can fit inside the channel along its transverse direction, whereas only one big square (but not two) is allowed to fit, i.e., $2.5=1+\sigma_{2} / \sigma_{1}<H / \sigma_{1}<$ $2 \sigma_{2} / \sigma_{1}=3$. In Sec. III B a mixture with $\sigma_{2} / \sigma_{1}=2$ is studied. This time configurations where one big plus one small, or again two big squares, are both forbidden, which is expressed by the condition $2<H / \sigma_{1}<1+\sigma_{2} / \sigma_{1}=3$.

\section{A. The $\sigma_{2} / \sigma_{1}=3 / 2$ mixture}

First we analyze the close-packing properties of the mixture. For composition $x \geqslant 3 / 5$ the close-packing configuration can be reached by adding up along the channel two kinds of clusters in close contact. Big clusters, $\mathcal{N}_{\mathrm{b}}$ in number, consist of groups of five particles: two big squares joined in the direction along the channel and in contact with one wall, and three small squares, also joined along the channel, located on top of (or below) the big squares and occupying the same length, parallel to the walls, as the big squares. The other, smaller clusters, $\mathcal{N}_{\mathrm{s}}$ in number, are made of small squares grouped together in dimers and consist of two squares, perfectly aligned along the transverse direction, each one in contact with opposite walls. See Fig. 1 for a sketch of a possible close-packing configuration. The number of clusters should fulfill the relation $3 \mathcal{N}_{\mathrm{b}}+2 \mathcal{N}_{\mathrm{s}}=\mathrm{x} \mathcal{N}$ and $2 \mathcal{N}_{\mathrm{b}}=$
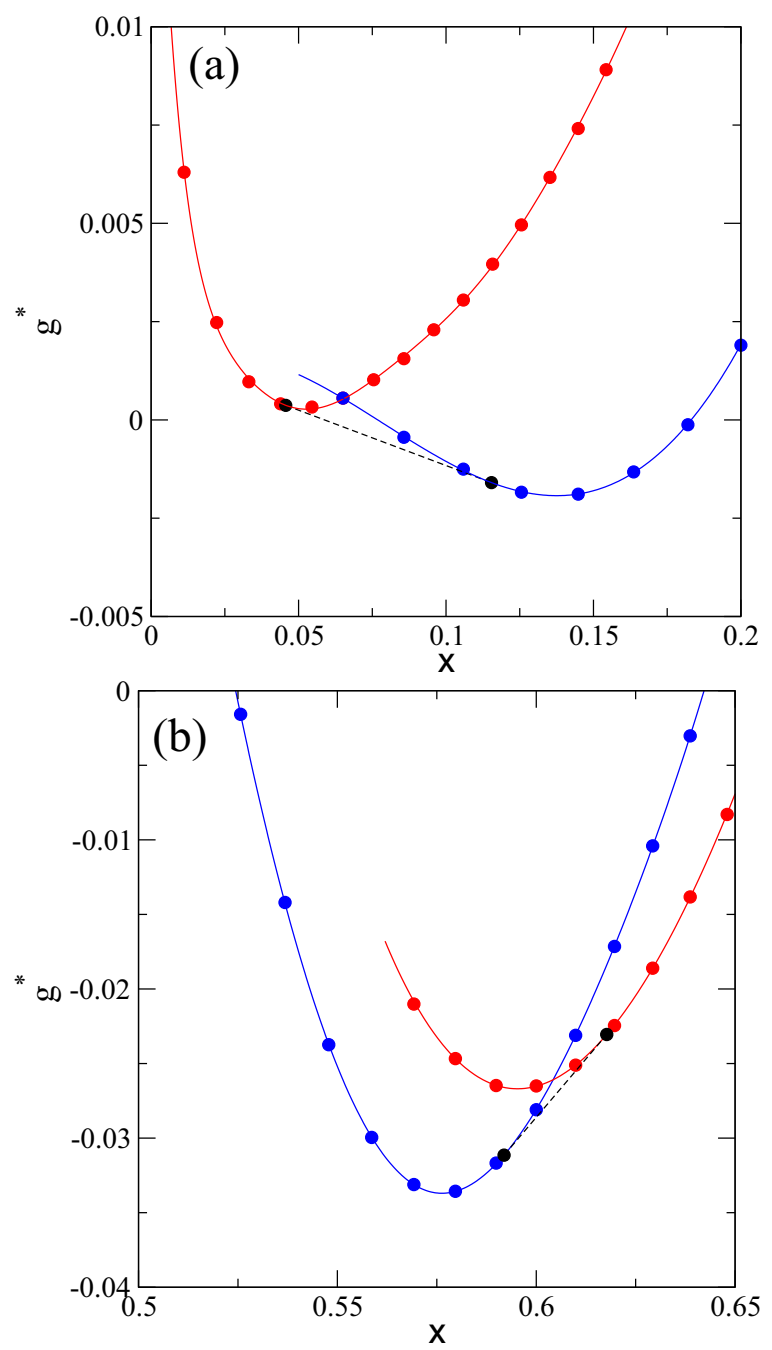

FIG. 3. Gibbs free energy per particle in reduced thermal units minus a straight line vs mean molar fraction $\mathrm{X}$, with (a) $g^{*} \equiv g-$ $17.856+13.184 \mathrm{x}$ and (b) $g^{*} \equiv g-15.959+8.873 \mathrm{x}$. Two different intervals of $\mathrm{X}$ are shown, located where transitions from symmetric (red curve) to asymmetric (blue curve) [shown in (a)] and from asymmetric to symmetric [shown in (b)] phases take place. Results correspond to a confined binary mixture of PHSs with $\sigma_{2} / \sigma_{1}=1.5$, $H / \sigma_{1}=2.6$, and $p_{0}^{*}=4$. Solid curves are least-squares polynomial fits to the red and blue symbols, which represent the calculated points. The coexisting points are indicated with black symbols joined by dashed lines.

$(1-\mathrm{x}) \mathcal{N}$ [and thus $\mathcal{N}_{\mathrm{b}}=(1-\mathrm{x}) \mathcal{N} / 2$ and $\mathcal{N}_{\mathrm{s}}=(5 \mathrm{x}-$ 3) $\mathcal{N} / 4$ ], with $\mathcal{N}$ the total number of particles. The packing fraction at close packing can be calculated as the ratio between the total area occupied by all clusters divided by the total area, i.e.,

$$
\eta_{\mathrm{CP}}=\frac{\mathcal{N}_{\mathrm{b}}\left(2 \sigma_{2}^{2}+3 \sigma_{1}^{2}\right)+2 \mathcal{N}_{\mathrm{s}} \sigma_{1}^{2}}{\left[3 \mathcal{N}_{\mathrm{b}} \sigma_{1}+\mathcal{N}_{\mathrm{s}} \sigma_{1}\right] H}=\frac{9-5 \mathrm{x}}{3-\mathrm{x}} \frac{\sigma_{1}}{H},
$$

for $x \geqslant 3 / 5$. For the case $x \leqslant 3 / 5$ the close-packing configuration can be reached by adding in close contact along the channel the same big clusters as defined previously, with a total number of $\mathcal{N}_{\mathrm{b}}$, and small clusters, with a total number of $\mathcal{N}_{s}$, this time formed by a single big square in any position 

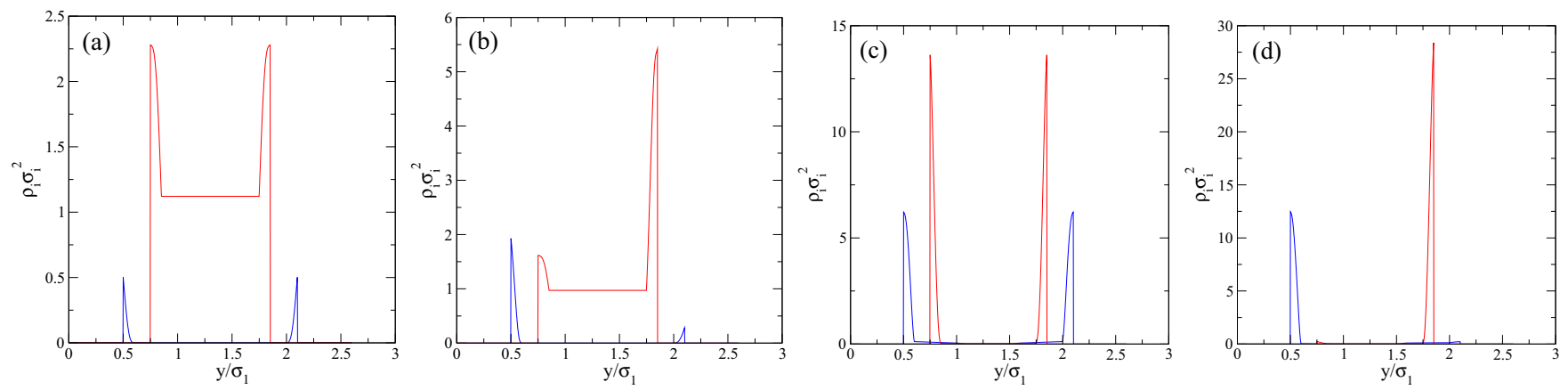

FIG. 4. Coexistence density profiles corresponding to the two first-order phase transitions shown in Fig. 3 for $\sigma_{2} / \sigma_{1}=1.5, H / \sigma_{1}=2.6$, and $p_{0}^{*}=4$ in (a) and (b) the low-molar-fraction region and (c) and (d) the high-molar-fraction region. (a) and (c) are symmetric phases, while (b) and (d) are asymmetric phases. Blue and red curves correspond to scaled density profiles $\rho_{1}(y) \sigma_{1}^{2}$ and $\rho_{2}(y) \sigma_{2}^{2}$ of small and big species, respectively.

along the transverse direction. The numbers $\left\{\mathcal{N}_{\mathrm{b}}, \mathcal{N}_{\mathrm{s}}\right\}$ fulfill $3 \mathcal{N}_{\mathrm{s}}=\mathrm{x} \mathcal{N}$ and $2 \mathcal{N}_{\mathrm{s}}+\mathcal{N}_{\mathrm{b}}=(1-\mathrm{x}) \mathcal{N}$ [and consequently $\mathcal{N}_{\mathrm{s}}=\mathrm{x} \mathcal{N} / 3$ and $\left.\mathcal{N}_{\mathrm{b}}=(3-5 \mathrm{x}) \mathcal{N} / 3\right]$. Then the packing fraction at close packing can be calculated for $x \leqslant 3 / 5$ as

$$
\eta_{\mathrm{CP}}=\frac{\mathcal{N}_{\mathrm{b}}\left(2 \sigma_{2}^{2}+3 \sigma_{1}^{2}\right)+\mathcal{N}_{\mathrm{s}} \sigma_{2}^{2}}{\left(3 \mathcal{N}_{\mathrm{b}} \sigma_{1}+\mathcal{N}_{\mathrm{s}} \sigma_{2}\right) H}=\frac{9-5 \mathrm{x}}{6(1-\mathrm{x})} \frac{\sigma_{1}}{H} .
$$

The function $\eta_{\mathrm{CP}}(\mathbf{x})$, given by Eqs. (20) and (21) for the case $H / \sigma_{1}=2.6$, is plotted in green in Fig. 2. The maximum packing fraction obviously corresponds to $x=3 / 5$ with $\eta_{\mathrm{CP}}^{(\max )}=\eta_{\mathrm{CP}}(3 / 5)=5 \sigma_{1} / 2 H$. The packing fractions for the one-component fluids composed of big and small particles are, respectively, $\eta_{\mathrm{CP}}(0)=3 \sigma_{1} / 2 H$ and $\eta_{\mathrm{CP}}(1)=2 \sigma_{1} / H$. In the same figure we plot the results from DFT calculations for the same pore width $H / \sigma_{1}=2.6$ at a fixed pressure $p_{0}^{*}=4$. Two different solutions are obtained, corresponding to two different local minima of the Gibbs free energy per particle. The red line represents the so-called symmetric solution, which has density profiles symmetric with respect to a line parallel to the $x$ axis that passes through the middle of the channel, i.e., $\rho_{i}(y)=\rho_{i}(H-y)$. The blue line, in contrast, represents an asymmetric solution, with $\rho_{i}(y) \neq \rho_{i}(H-y)$. Note that the asymmetric solution only exists in a particular interval of molar fractions, whereas the symmetric profile exists for all $\mathbf{x}$. The former gives a higher value of mean packing fraction $\eta$ (since the blue curve is above the red one). Both curves have their maxima located close to $x \approx 3 / 5$, where the maximum value at close packing is reached. The confined mixture exhibits two first-order phase transitions that take place as the molar fraction is increased from 0 to 1 . Both the symmetric to asymmetric and the asymmetric to symmetric transitions are correspondingly labeled in Fig. 2, with the coexisting values shown by two pairs of red and blue circles.

The asymmetric phase is stable in an interval of $x$ between the blue circles of Fig. 2. This can be concluded from Fig. 3, where we plot the Gibbs free energy per particle for two different ranges of $x$ [Figs. 3(a) and 3(b)] located close to both phase transitions. In both cases straight lines have been subtracted to improve visualization. The circles correspond to values of $\mathrm{x}$ where DFT calculations were performed, and the pressure was set equal to $p_{0}^{*}=4$. Red and blue curves are polynomial fits of the symmetric and asymmetric solutions, respectively, which were used to calculate coexistence through a double-tangent construction. We checked that the energy $g$ of the asymmetric phase is always below that of the symmetric phase in the interval $x \in[0.2,0.5]$. The four coexisting density profiles are shown in Fig. 4. Their symmetric or asymmetric character is quite apparent. In the asymmetric phase big and small squares are preferentially absorbed at different walls, a type of microsegregation transition. In contrast, in the symmetric phase both species are equally adsorbed at both walls.

The driving force for microsegregation is entropy. It is clear that, at close packing, two possible configurations of big clusters in the symmetric phase are equally represented, i.e., big clusters containing big squares in contact with different walls are equally likely. In contrast, in the asymmetric phase this symmetry is broken, with one of the configurations overrepresented with respect to the other. Close packing can be attained by both asymmetric and symmetric phases, but the latter is more disordered in terms of mixing entropy and consequently has a lower free energy. However, far from close packing, when pressure is not too large, e.g., $p_{0}^{*}=4$, the situation can be different. Since big squares will be alternatively absorbed at both walls in the symmetric phase while the space between big squares in contact with the same wall is moderately filled with small squares, it is clear that it is not possible for big squares to overpass each other: The motion of small squares along the $x$ axis is severely restricted due to the jammed configuration of large particles. Thus the configurational entropy, related to the total number of allowed particle configurations, drops and consequently the free energy increases as compared to that of the quasiperfect asymmetric phase. In the latter big squares are not jammed (since most of them are adsorbed at the same wall) and therefore particles can move along the channel with much more freedom (the only constraint being hard-core interactions with the lateral neighbors). Of course particles can also move along the $y$ axis, but they have similar freedom in both phases.

We performed coexistence calculations for several values of pressure to construct a phase diagram for $H / \sigma_{1}=2.6$. This is shown in Fig. 5, in the $p^{*}-\mathrm{X}$ and $\eta$-X planes. We see that the asymmetric stability region is laterally bounded (in the $\mathrm{X}$ direction) by first-order symmetric to asymmetric and asym- 

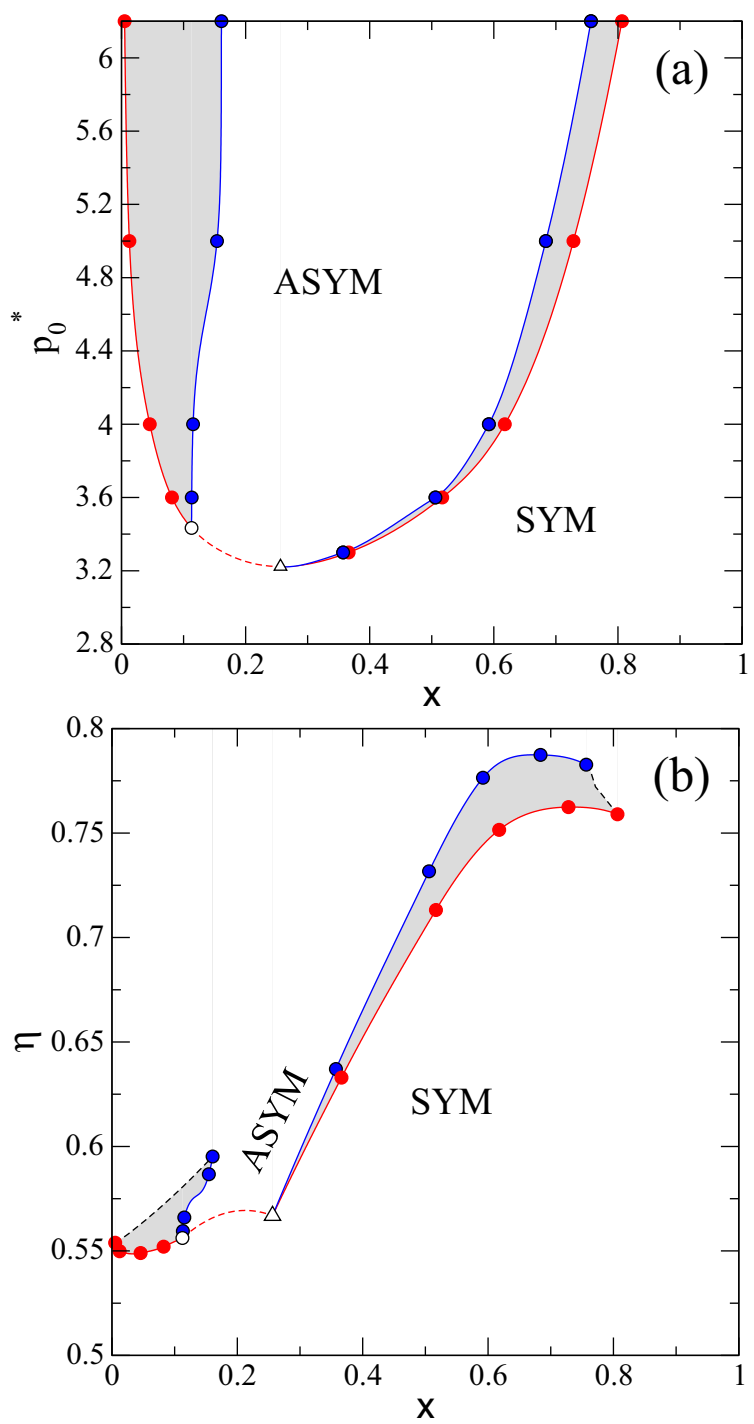

FIG. 5. Phase diagrams of a confined binary mixture of PHSs with $\sigma_{2} / \sigma_{1}=1.5$ and $H / \sigma_{1}=2.6$ : (a) $p_{0}^{*}$ vs $\mathrm{X}$ and (b) $\eta$ vs X. Red and blue closed circles represent the coexisting values of the symmetric and asymmetric states, respectively. The open circle represents the left tricritical point separating the coexisting binodals (solid lines) from the continuous-phase-transition curve (dashed line). The open triangle represents the right tricritical point.

metric to symmetric transitions lines. At low pressures the symmetric to asymmetric transition terminates in a left tricritical point (open circle). From this point the transition becomes continuous. This line meets the binodals of the asymmetric to symmetric transition at the right tricritical point (open triangle). Note the strong fractionation of the symmetric to asymmetric transition: The compositions of the coexisting phases are very different from those of the asymmetric to symmetric transition. As more packed configurations are reached by increasing the amount of small squares, the phase diagram in the $\eta$-X plane [Fig. 5(b)] becomes highly asymmetric, i.e., there is a large difference in packing fraction values of the coexistence binodals to the left and right of the end critical point.

The phase diagram for a wider pore width of $H / \sigma_{1}=2.8$, shown in Fig. 6, was also calculated. In wider pores the
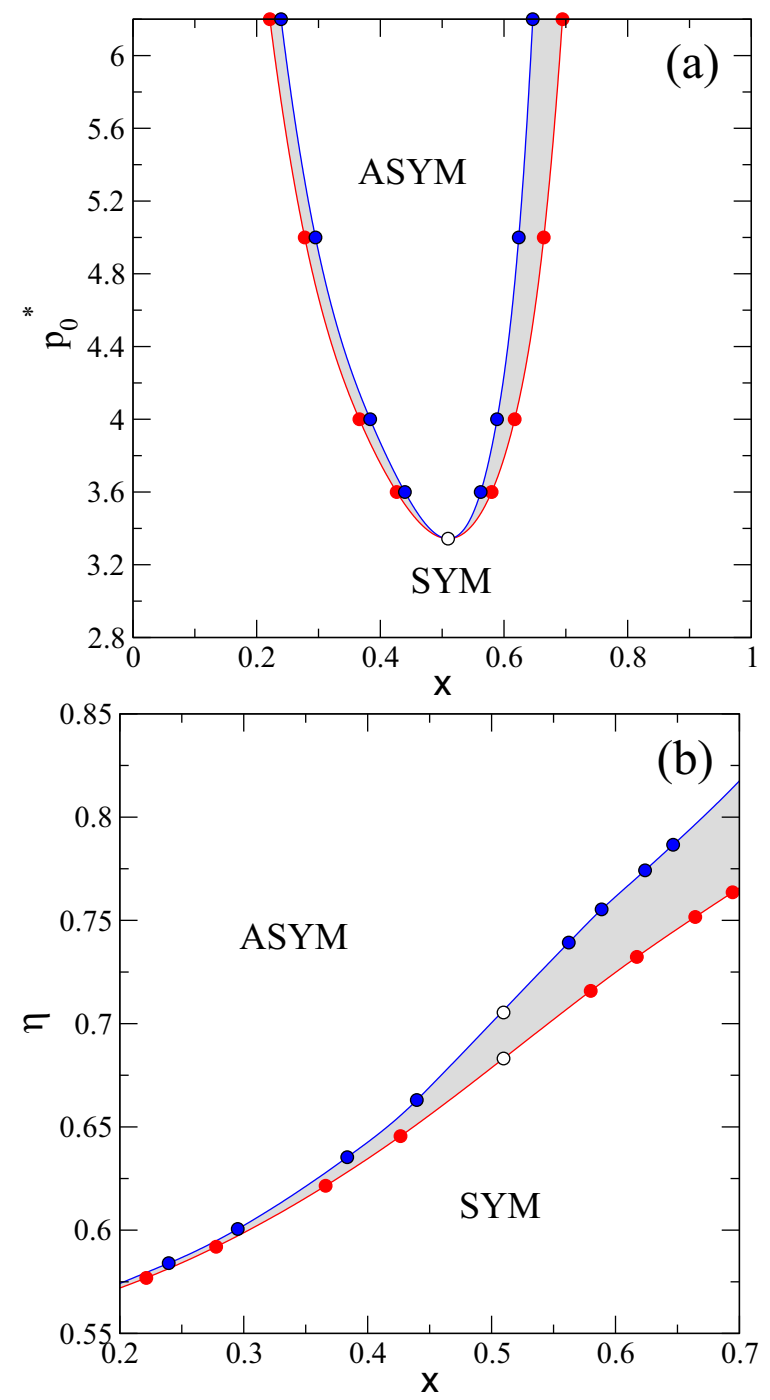

FIG. 6. Phase diagrams for the confined binary mixture of PHSs with $\sigma_{2} / \sigma_{1}=1.5$ and $H / \sigma_{1}=2.8$ : (a) $p_{0}^{*}$ vs $\mathbf{x}$ and (b) $\eta$ vs $\mathbf{x}$. Red and blue closed circles represent the coexisting values corresponding to symmetric and asymmetric states, respectively. The open circle indicates the azeotropic point.

entropically driven microsegregation, resulting from particlemotion restrictions in jammed symmetric configurations, still operates, but to a lesser extent because the transverse spatial freedom of particles increases with $H / \sigma_{1}$. Thus the ratio between the gain in lateral free length (resulting from microsegregation) and the transverse free length is lower. We should recall that configurational entropy competes with mixing entropy (which prevents microsegregated states). As a final result the region of asymmetric-phase stability in the $p_{0}^{*}$ - phase diagram shrinks with $H / \sigma_{1}$, a fact that can be confirmed by looking at Fig. 7(a). It can be seen that for the highest pressure used $\left(p^{*}=6.2\right)$ the stability interval in $\mathbf{X}$ of the asymmetric phase is now $\sim[0.23,0.65]$, which is smaller than $[0.15,0.75]$ (which corresponds to the $H / \sigma_{1}=2.6$ case). Another interesting feature of the phase diagram is the weaker character of the first-order symmetric to asymmetric transition to the left of the azeotropic point (open circle). The azeotropic 

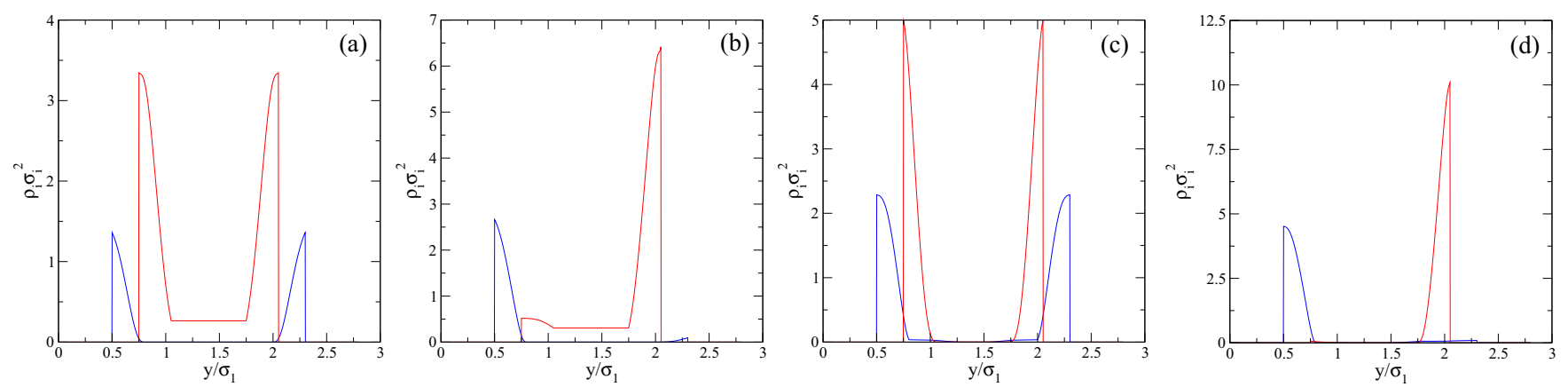

FIG. 7. Coexistence density profiles corresponding to the two first-order phase transitions shown in Fig. 6 for $\sigma_{2} / \sigma_{1}=1.5, H / \sigma_{1}=2.8$, and $p_{0}^{*}=4$ in (a) and (b) the low-molar-fraction region and (c) and (d) the high-molar-fraction region. (a) and (c) are symmetric phases, while (b) and (d) are asymmetric phases. Blue and red curves correspond to scaled density profiles $\rho_{1}(y) \sigma_{1}^{2}$ and $\rho_{2}(y) \sigma_{2}^{2}$ of small and big species, respectively.

character of the latter can be inferred from Fig. 7(b), which demonstrates the existence of a coexisting gap in $\eta$ at this point, despite the fact that the composition of the coexisting phases is the same. The binodals are monotonically increasing functions of $\mathbf{x}$, showing the higher packing inside the pore resulting from an increase in the number of small squares.

Figure 7 depicts the four coexisting density profiles for this pore width and with pressure set equal to $p_{0}^{*}=4$. The following results can be extracted: (i) Density profiles are broadened compared to those for the thinner pore and (ii) adsorption of big squares at the walls is increased; the heights of the central plateau in the density profile $\rho_{2}(y)$ [see Figs. 7(a) and 7(b)] are lower than those of Figs. 2(a) and 2(b). This effect can be understood in terms of the low values of coexisting compositions for the symmetric to asymmetric transition in the thin pore: There exists a large number of big squares which do not contribute to the formation of the big clusters and they freely fluctuate between both walls.

The decrease in asymmetric phase stability with pore width $H / \sigma_{1}$ at a fixed pressure (in particular for $p_{0}^{*}=4$ ) is confirmed in Fig. 8(a), where we plot the interval $\Delta \mathbf{x}=\mathbf{x}^{a, 2}-\mathbf{x}^{a, 1}$ [with $\mathrm{X}^{a, i}$ the coexisting values of the left $(i=1)$ and right $(i=2)$ asymmetric binodals] in which stable asymmetric solutions are found vs the free transversal length $\left(H-\sigma_{1}-\sigma_{2}\right) / \sigma_{1}$. Also plotted is the difference in packing fractions at those points $\left(\Delta \eta=\eta^{(a, 2)}-\eta^{(a, 1)}\right)$, which does not change much but exhibits a maximum.

As mentioned before, the entropic mechanism that drives microsegregation at finite pressure does not operate at close packing because in this case (infinite pressure) the lateral free length that allows particle motion is absent, while mixing entropy favors the formation of symmetric states. Therefore, at very high pressure, an upper azeotropic point is expected in the phase diagram: We conjecture the existence of a finite region in the phase diagram where a reentrant asymmetric phase is stable. An indication that this could certainly be the case can be seen in Fig. 8(b), where we plot the EOS of the symmetric (red solid line) and asymmetric (blue solid line) phases for fixed values of compositions, $x=0.36611$ and $x=0.38352$, respectively. These are the coexisting values of the symmetric to asymmetric transition at $p_{0}^{*}=4$. The symmetric and asymmetric phases are stable in the intervals $0<p_{0}^{*}<4$ and $4<p_{0}^{*} \lesssim 9$, respectively. Note how the EOS of a symmetric phase with a fixed composition $\mathrm{x}=0.38352$ (dashed red line) intersects the blue solid curve, which indicates that for pressures $p^{*} \gtrsim 9$ the asymmetric phase might lose stability with respect to the symmetric phase. Unfortunately our numerical scheme to implement the DF minimization becomes unstable at these high pressures, and an alternative method, such as a density-profile parametrization, is needed to validate this conjecture.

\section{B. The $\sigma_{2} / \sigma_{1}=2$ mixture}

To find the close-packing configurations for $\sigma_{2} / \sigma_{1}=2$ we apply the same reasoning as before: The close-packed limit can be reached by joining $\mathcal{N}_{\mathrm{b}}$ big clusters (constituting a single big square) with $\mathcal{N}_{\mathrm{s}}$ small clusters (formed by dimers of small squares, perfectly aligned along $y$ ). The total area occupied by both clusters is $\mathcal{N}_{\mathrm{b}} \sigma_{2}^{2}+2 \mathcal{N}_{\mathrm{s}} \sigma_{1}^{2}$, whereas the total occupied length along the channel is $\mathcal{N}_{\mathrm{b}} \sigma_{2}+\mathcal{N}_{\mathrm{s}} \sigma_{1}$. As the numbers $\left\{\mathcal{N}_{\mathrm{b}}, \mathcal{N}_{\mathrm{s}}\right\}$ fulfill the condition $\mathcal{N}_{\mathrm{b}}=(1-\mathrm{x}) \mathcal{N}$ and $\mathcal{N}_{\mathrm{s}}=\mathrm{x} \mathcal{N} / 2$, we arrive at

$$
\eta_{\mathrm{CP}}=\frac{\mathcal{N}_{\mathrm{b}} \sigma_{2}^{2}+2 \mathcal{N}_{\mathrm{s}} \sigma_{1}^{2}}{\left(\mathcal{N}_{\mathrm{b}} \sigma_{2}+\mathcal{N}_{\mathrm{s}} \sigma_{1}\right) H}=\frac{(1-\mathbf{x})\left(\sigma_{2} / \sigma_{1}\right)^{2}+\mathbf{x}}{2(1-\mathbf{x}) \sigma_{2} / \sigma_{1}+\mathrm{x}} \frac{2 \sigma_{1}}{H}=\frac{2 \sigma_{1}}{H} .
$$

The close-packing value does not depend on composition. In Fig. 9(a) these limits are shown for $H / \sigma_{1}=2.2,2.4$, and 2.6. Also the functions $\eta(\mathbf{x})$ are plotted for the same values of pore widths as obtained from the DF minimization, by setting the pressure equal to $p_{0}^{*}=5$. Clearly, the packing fractions, which are monotonically decreasing functions of $\mathbf{x}$, do not change too much with composition as compared to the case $\sigma_{2} / \sigma_{1}=$ 1.5. The intervals of $x$ between the closed circles represent the instability region in mixture composition with respect to demixing transitions. This behavior can be confirmed by plotting the Gibbs free energy per particle (subtracting a straight line) $g^{*}$ vs $\mathbf{X}$, as we do in Fig. 9(b) for $H / \sigma_{1}=2.4$ and $p_{0}^{*}=5$. Strong demixing between two confined phases, each one rich in one of species, is confirmed. The phase separation has a clear lateral symmetry, i.e., both phases are separated along the channel with a Gibbs-dividing interface perpendicular to the channel. This is a kind of macrosegregation, completely different from the microsegregation obtained 

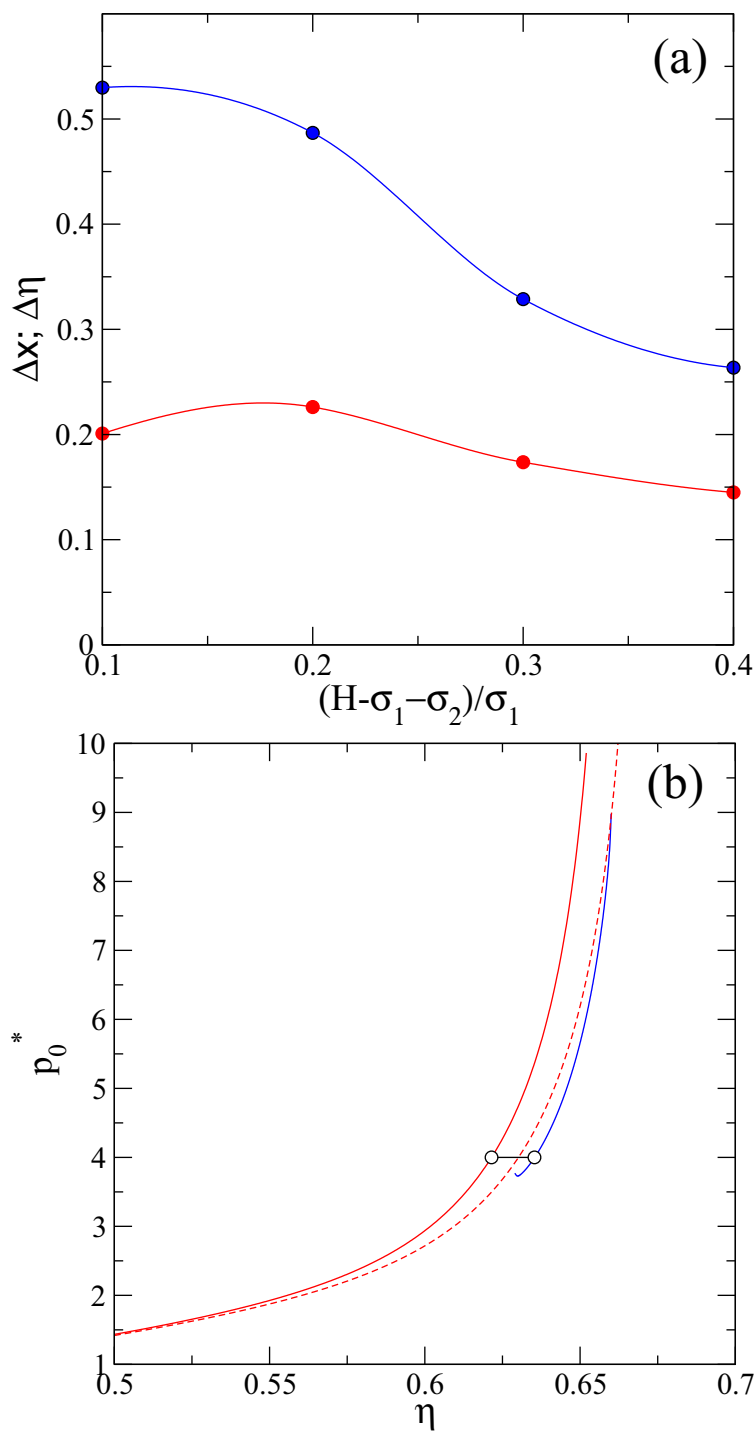

FIG. 8. (a) Differences in coexisting molar and packing fractions of asymmetric phases $\Delta \mathbf{x} \equiv \mathbf{x}^{(a, 2)}-\mathbf{x}^{(a, 1)}$ (blue) and $\Delta \eta \equiv$ $\eta^{(a, 2)}-\eta^{(a, 1)}$ (red), as functions of the scaled free length $\left(H-\sigma_{1}-\right.$ $\left.\sigma_{2}\right) / \sigma_{1}$, for a binary mixture with $\sigma_{2} / \sigma_{1}=1.5$ and pressure $p_{0}^{*}=5$. (b) Equation of state of a confined binary mixture with $\sigma_{2} / \sigma_{1}=$ 1.5 and $H / \sigma_{1}=2.8$. The red and blue solid lines correspond to symmetric and asymmetric states, with compositions set equal to their corresponding coexisting values at $p_{0}^{*}=4$, i.e., $\mathbf{x}=0.36611$ and $x=0.38352$, respectively. Coexisting states are indicated by open circles. The EOS corresponding to a symmetric phase with $x=0.38352$ is shown by a dashed red curve. Note that this curve intersects the blue line at high pressures, indicating that the asymmetric states will become unstable and an upper azeotropic point probably exists in the phase diagram.

before for the case $\sigma_{2} / \sigma_{1}=1.5$. However, the density profiles are now symmetric always.

To find the phase diagram, we have calculated the coexisting values of $\mathbf{x}$ and $\eta$ for a set of different values of $p_{0}^{*}$ and for a fixed pore width $H / \sigma_{1}=2.4$, via the doubletangent construction of $g(\mathbf{x})$. Phase diagrams in $p^{*}-\mathbf{x}$ and $\eta-\mathbf{x}$ coordinates are plotted in Figs. 10(a) and 10(b), respectively. Dashed lines in Fig. 10(b) correspond to different isobars
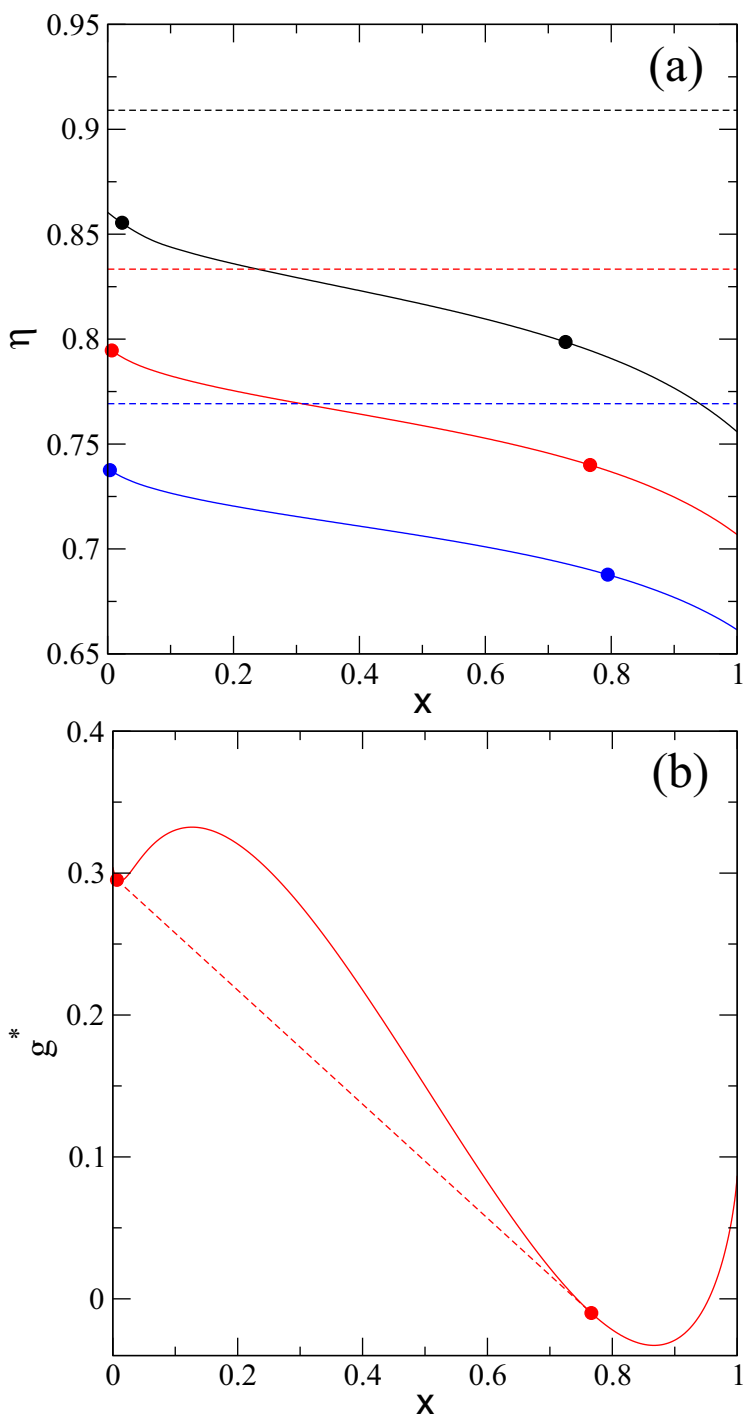

FIG. 9. (a) Packing fraction vs molar fraction for a confined binary mixture of PHSs with $\sigma_{2} / \sigma_{1}=2, p_{0}^{*}=5$, and $H / \sigma_{1}=2.2$ (black solid line) 2.4 (red solid line), and 2.6 (blue solid line). Close-packing values $\eta_{\mathrm{CP}}$ for the same values of $H / \sigma_{1}$ are shown by dashed lines. (b) Scaled Gibbs free energy per particle minus a straight line $g^{*} \equiv \beta g-27.232+18.124 \mathrm{x}$ vs molar fraction for the same mixture and for $H / \sigma_{1}=2.4$. The closed circles joined with a dashed line indicate the coexistence values of $\mathbf{x}$.

inside the demixed region. The phase separation ends in a critical point (open circle), below which the mixture is stable. As pressure is increased from that point, the coexisting phases become more similar to the confined one-component fluids. As an example of coexisting phases, Fig. 11 shows the density profiles of small and big squares for the low-X [Fig. 11(a)] and large-X [Fig. 11(b)] coexisting phases, with $p_{0}^{*}=4$ and $H / \sigma_{1}=2.4$. Note that in Fig. 11(a) the density profile of small species is not visible at the scale of the figure, demonstrating the quasi-one-component character of the mixture. We can see in Fig. 11(b) that, while big squares always fluctuate close to the center of the pore, small squares are strongly adsorbed at both walls. 

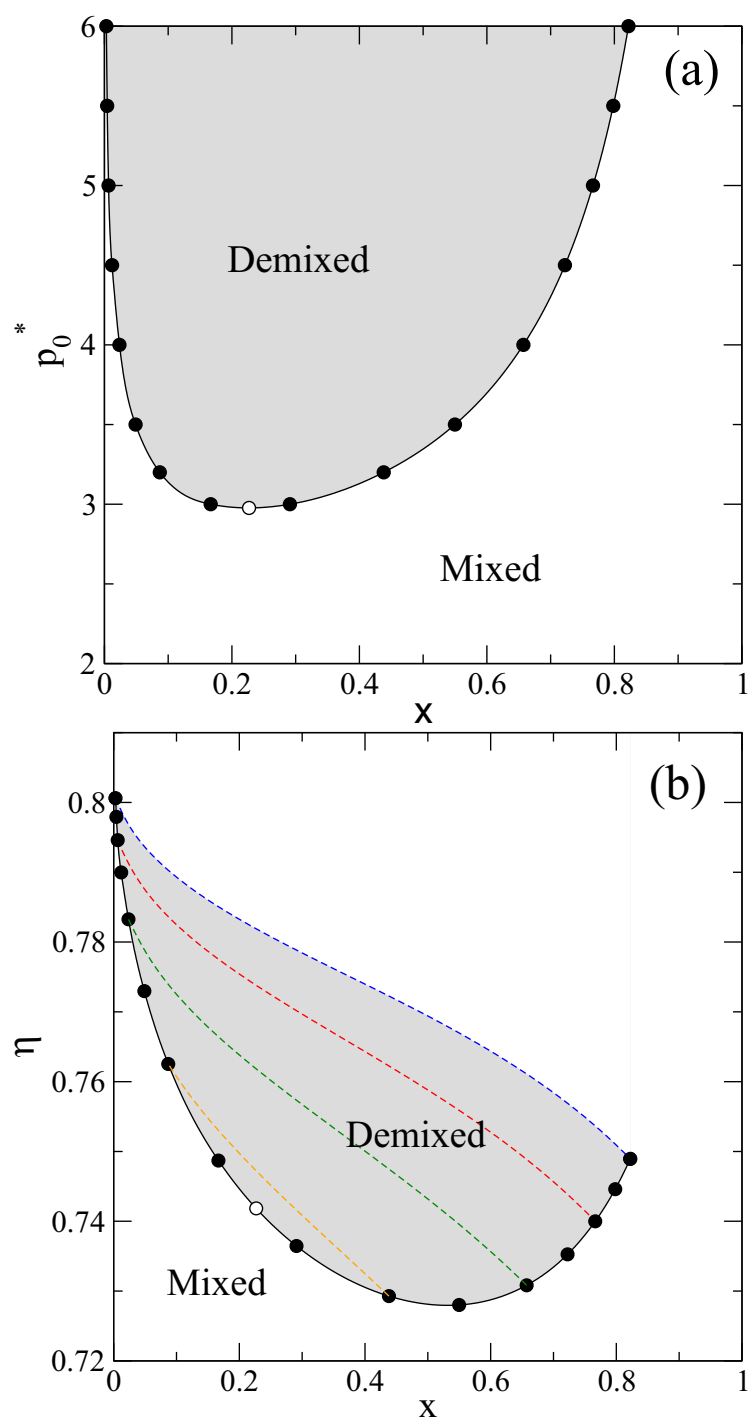

FIG. 10. Phase diagrams in the (a) pressure-composition plane and (b) packing-fraction-composition planes of a confined binary mixture of PHSs with $\sigma_{2} / \sigma_{1}=2$ and $H=2.4$. In (b) four isobars, for $p_{0}^{*}=6$ (blue), 5 (red), 4 (green), and 3.2 (orange), are shown with dashed lines.

The phase separation once again is related to entropy. When both species are mixed, e.g., when clusters formed by dimers of small squares are surrounded by big squares, lateral motion of small particles is strongly restricted because small and big species cannot overpass each other. Also, if one dimer of small particles is located between two big squares, the motion of these highly constrained small squares entails the breaking of dimers, with a lowering in the local packing fraction. When the mixture is well separated, small squares can move in the lateral direction much more freely because the presence of other small particles in front does not constrain their motion. Thus, the dimers can be continuously formed and destroyed without altering the local packing of particles.

An interesting issue is how the demixing transition depends on pore width $H$. The answer to this question is given by Fig. 12, where the demixing gaps in $\mathrm{X}$ (black lines) and $\eta$ (red lines) are plotted as a function of the free length
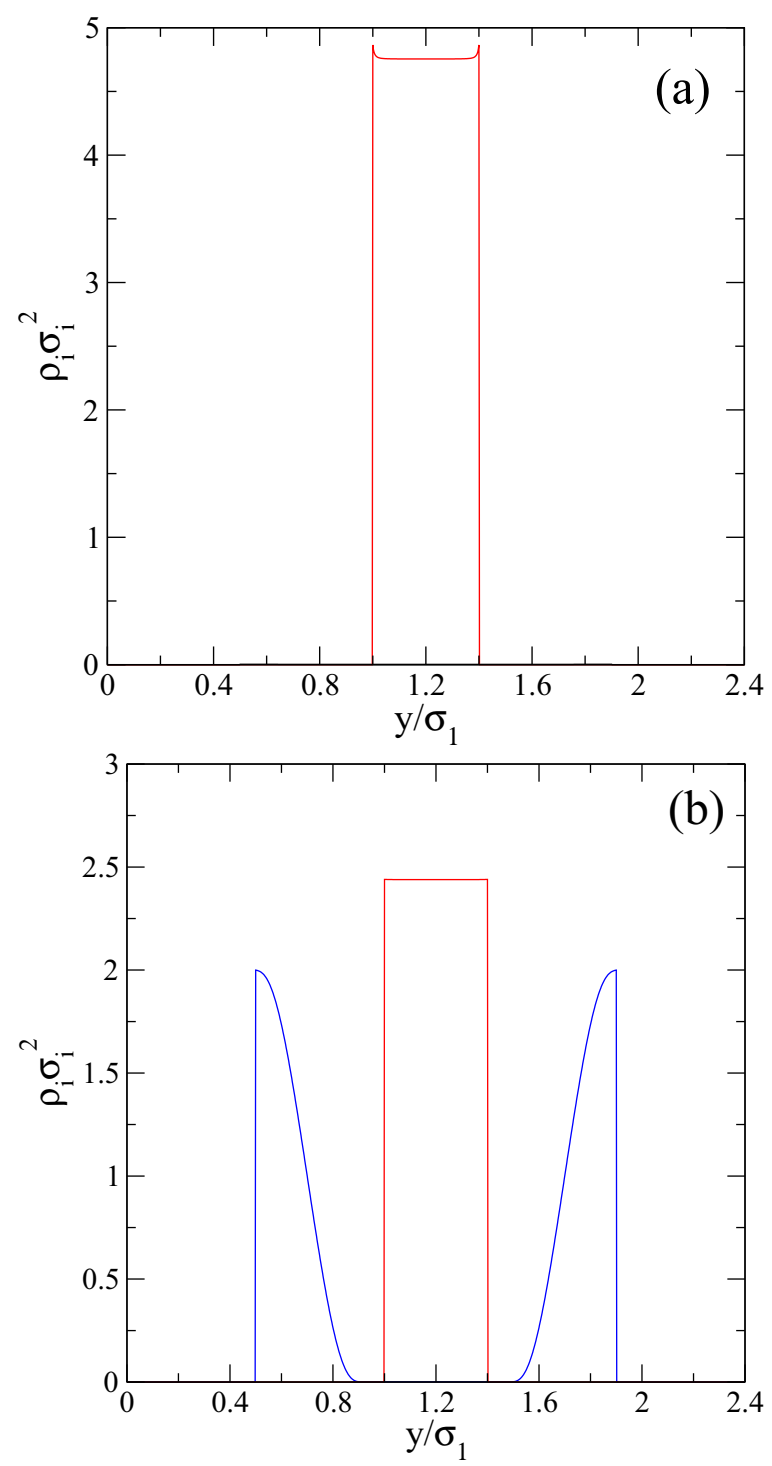

FIG. 11. Density profiles of a confined binary mixture with $\sigma_{2} / \sigma_{1}=2, H / \sigma_{1}=2.4$, and $p_{0}^{*}=5$ corresponding to the coexisting phases with (a) low and (b) high molar fractions.

$\left(H-\sigma_{2}\right) / \sigma_{1}$ for a fixed pressure $p_{0}^{*}=5$. As the pore becomes wider the demixing, in terms of fractionation, is stronger $\left(\Delta \mathbf{x} \equiv \mathbf{x}^{(2)}-\mathbf{x}^{(1)}\right.$ is an increasing function of $\left.H\right)$, while the gap in packing fraction decreases. The latter result is expected because packing of squares inside the pore is less effective as the pore becomes wider, so the two coexistence values of $\eta$ decrease, to such an extent that the difference $\Delta \eta \equiv \eta^{(2)}-$ $\eta^{(1)}$ is a monotonically decreasing function of $\mathrm{x}$. However, the relative gap $\Delta \eta / \eta^{(1)}$ turns out to be constant with a value close to 0.07 . This interesting trend, namely, a stronger demixing as $H$ increases, is opposite to that obtained for the $\sigma_{2} / \sigma_{1}=1.5$ mixture. As shown in the preceding section, the microsegregation transition is enhanced when the pore becomes narrower.

To end this section, we comment on the relation between the phase behavior of the confined system and that of a similar system subject to periodic boundary conditions (PBCs). To investigate this, we have imposed the conditions $\rho_{i}(y+H)=$ 


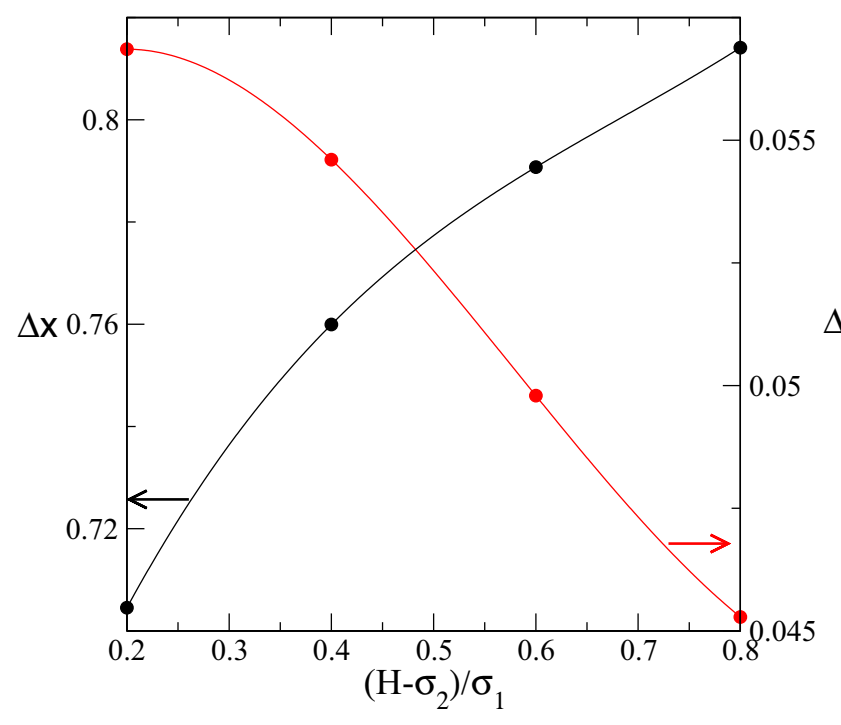

FIG. 12. Differences in the coexisting molar fraction $\Delta \mathrm{x}=$ $\mathbf{x}^{(2)}-\mathbf{x}^{(1)}$ (black) and packing fraction $\Delta \eta=\eta^{(2)}-\eta^{(1)}$ (red) of the demixed phases as functions of the free length $\left(H-\sigma_{2}\right) / \sigma_{1}$ for a binary mixture of confined PHSs with $\sigma_{2} / \sigma_{1}=2$ and $p_{0}^{*}=5$.

$\rho_{i}(y)$ on the density profiles, focusing on the binary mixture with $\sigma_{2} / \sigma_{1}=2$. The period $H$ was chosen to be equal to the pore width of one of the mixtures analyzed previously, i.e., $H / \sigma_{1}=2.4$. We did not minimize the DF with respect to $H$, with the aim of making the comparison of the two results meaningful. Consequently, the phase diagram presented below is not the bulk one. Note that PBCs are normally used to mimic bulk phase behavior when the system is infinite in the $y$ direction, with the inhomogeneous phase being periodic in the same direction. However, the same condition can also describe a finite system of dimension $H$ in the $y$ direction. Unfortunately, the DF is unable to distinguish both situations, which is a strong drawback of this theoretical tool. Obviously, if a DF based on the two-body probability density, instead of the one-body density, could be constructed, it would certainly contain information on the finiteness of the system along $y$. Therefore, at present, results from the (one-body densitybased) DF and the TMM applied to the study of systems with PBC cannot be compared [48].

Figure 13 shows the phase diagram as obtained from DF minimization. The dashed lines represent continuous transitions between a fluid of PHSs and a periodic columnar phase with period $H / \sigma_{1}=2.4$. The latter was calculated by searching for the divergence of the structure-factor inverse matrix, as described in Appendix B. The solid lines (which join the calculated points) are the coexisting binodals of the demixing transitions. For relatively high composition $x \gtrsim 0.7$ and fixed pressure $p_{0}^{*}=4$, we find that the stable phase is the so-called $C_{1}$ columnar phase, formed by two layers of small squares (of period $d \equiv H / 2=1.2 \sigma_{1}$ ) where the centers of mass of big squares occupy interstitial positions between the layers and the density profiles of small and big squares having a phase difference of $d / 2$. [See Fig. 14(a), where these density profiles are plotted, and Fig. 15(a) for a sketch of particle configurations.] Note that big squares intersect the two adjacent layers formed by small squares. As $\mathbf{X}$ is decreased this phase loses
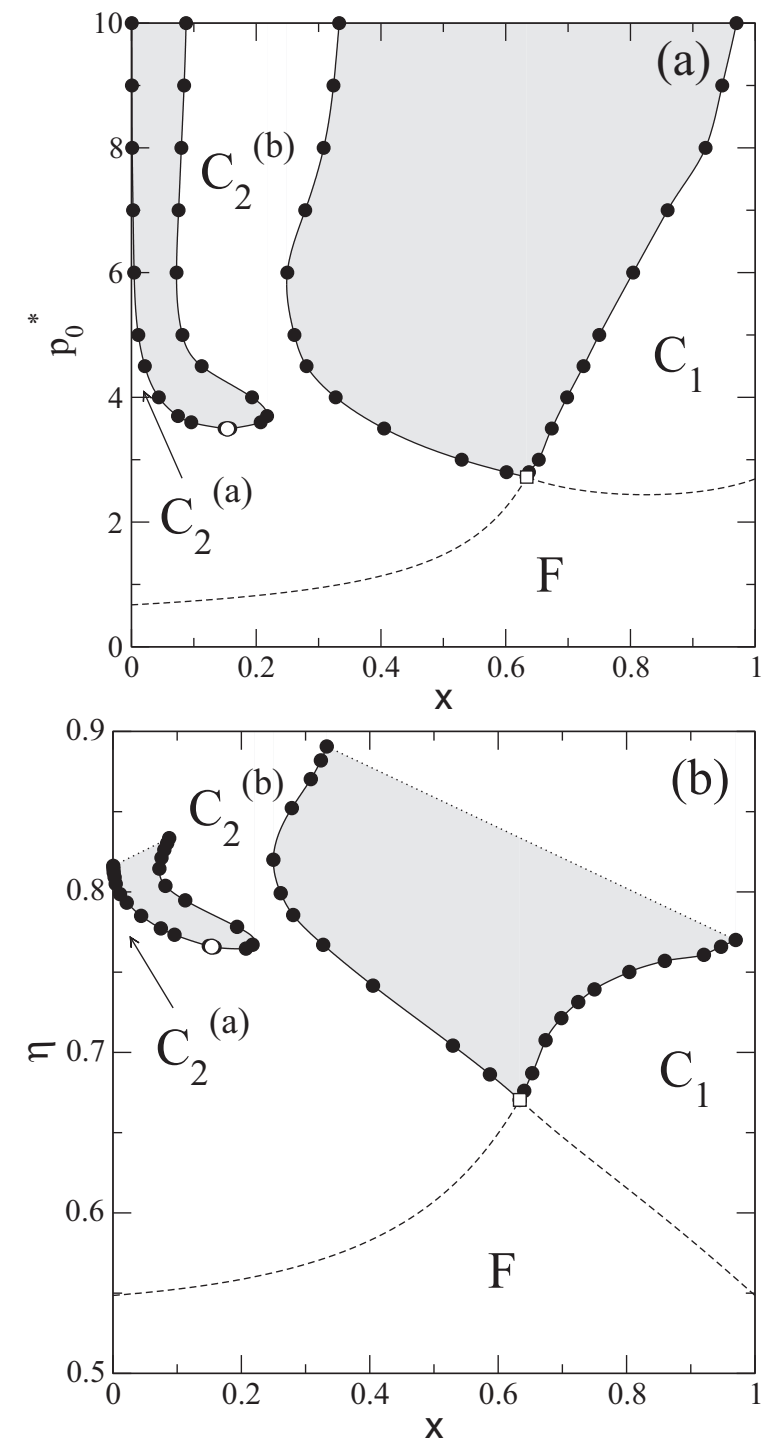

FIG. 13. (a) Phase diagram $p_{0}^{*}-\mathrm{x}$ of a binary mixture of PHSs with $\sigma_{2} / \sigma_{1}=2, H / \sigma_{1}=2.4$, and PBCs. Solid lines represent the coexisting binodals, whereas dashed lines indicate continuous phase transitions. Regions of stability of fluid (F) and different columnar $C_{i}^{(\alpha)}$ phases are correspondingly shown. (b) Same phase diagram as in (a), but in the $\eta$-X plane. Closed circles show calculated binodal points, the open circle shows the critical point, and the open square shows the tricritical point.

stability at $\mathrm{X} \sim 0.7$ and the system exhibits strong demixing to the so-called $C_{2}^{(b)}$ columnar phase, with a composition $\mathrm{x} \sim 0.3$ and formed by layers of big squares with small squares mostly microsegregated at the interstitials [see Fig. 14(b) for the density profiles and Fig. 15(b) for a sketch of particle configurations]. Now the periodicity is $d=H$. By further decreasing $\mathbf{x}$ it is found that this phase is stable up to $\mathrm{X} \sim 0.2$, where a new phase transition takes place to the so-called $C_{2}^{(a)}$ phase. This is very similar in structure to the $C_{2}^{(b)}$ phase, but the former exhibits a domedlike density profile for the big squares [see Fig. 14(c)], while the latter has the usual sharply peaked form [see Fig. 14(d)], with a small number of small squares located at the interstitials. The $C_{2}^{(a)}-C_{2}^{(b)}$ and $C_{1}-C_{2}^{(b)}$ transitions 

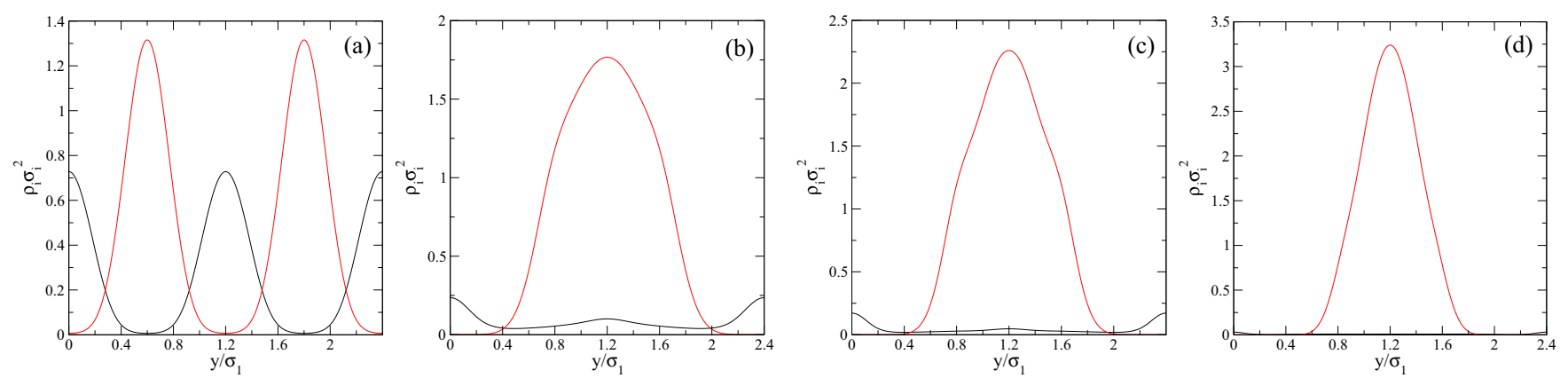

FIG. 14. Coexisting density profiles of a binary mixture with $\sigma_{2} / \sigma_{1}=2, H / \sigma_{1}=2.4, p_{0}^{*}=4$, and PBCs. From (a) to (d) density profiles correspond to $\mathbf{X}^{(1)}>\mathbf{X}^{(2)}>\mathbf{X}^{(3)}>\mathbf{X}^{(4)}$, i.e., the coexistence values of molar fractions for both demixing transitions found in the phase diagram of Fig. 13 at the corresponding pressure.

end in critical and tricritical points, respectively. The main conclusion drawn from these results is that scenarios of strong demixing and microsegregation are also present in a PHS fluid subject to PBCs. This is an indication that the bulk phase diagram will also contain these two features.

Crystalline phases (where both density profiles depend on both spatial coordinates) were not included in our study. At high pressure crystals will certainly become more stable than the exotic one-dimensional profiles we have found in the region of stability of $C_{2}^{(b)}$ at very high pressures (not shown here).

\section{CONCLUSION}

We have used the DF formalism, based on the FMT, to study the packing properties of extremely confined mixtures of PHSs in a slit pore. Two types of mixtures have been analyzed in detail by appropriately choosing particle sizes and pore width. In the first study, parameters were tuned to avoid configurations where two big squares are located opposite to each other while dimers of one big and one small or two small squares, but not three of them, can fit into the channel. In the second study parameters were arranged so as to avoid dimers formed by one big and one small square to fit into the channel, while two small squares can fit. We have shown that the theory predicts micro- and macrosegregation phase transitions for the first and the second mixture, respectively. Using the Gibbs free-energy potential for a set of fixed pressures, coexisting packing and molar fractions were calculated via a doubletangent construction. Thus the first-order character of phase

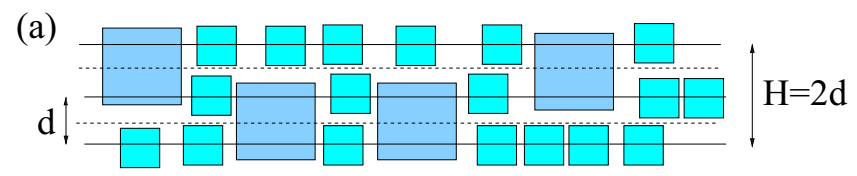

(b)

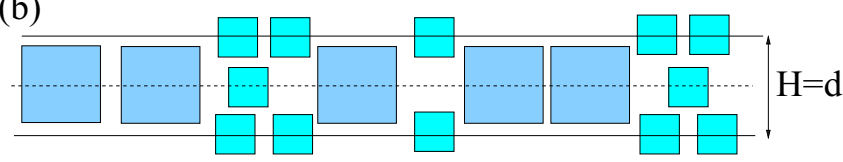

FIG. 15. Schematic of particle configurations of two different columnar phases found in the phase diagram with PBCs: (a) $C_{1}$ and (b) $C_{2}^{(a, b)}$. transitions at most pressures was identified and boundaries of stability regions for mixed and micro (macro)-demixed states were traced out. All phase transitions found have an entropic character, which is ultimately related to the jammed configurations of particles. These configurations arise when two big squares are close to each other and, at the same time and for the first mixture, they are symmetrically adsorbed at both walls. The jammed configurations severely restrict lateral motion of small particles (thus decreasing the configurational entropy) and these can explore a limited space as compared to that in macro- or microsegregated mixtures. Finally, by imposing PBCs we showed that demixing transitions between different columnar phases also take place in systems without external potentials restricting particle positions. In this case all demixed phases found also have a microsegregated structure, with one of the species forming the main columns and the other occupying the interstitial regions.

A comment on the real nature of phase transitions obtained here for the confined system is in order. Exact calculations using the TMM for one-component hard disks, squares, rectangles, or rhombuses confined in a slit geometry, with at most two layers of particles, show that these $(1+\epsilon)$-dimensional systems do not exhibit true phase transitions [34,35,37,38]. However their structural properties can dramatically change as pressure is increased. This behavior is usually associated with a peculiar shape of the EOS which, under certain conditions, contains a plateaulike segment and a corresponding sharp peak is visible in the heat capacity. With these results in mind, our analysis based on the (mean-field) DFT suggests that changes in particle configurations, driven by entropic forces, are adequately described by the theory, while the corresponding phase transitions are not. Our claim is that, for high enough pressures, the two confined mixtures studied here will contain an important number of large micro- and macrosegregated clusters, respectively. Although these clusters can symmetrically adopt two configurations in the case of the first mixture studied, their presence can be confirmed by calculating the two-body particle correlation function using the TMM.

The most important result from our mean-field model, as applied to the second confined mixture (with $\sigma_{2} / \sigma_{1}=2$ ), consists in the prediction of a lateral demixing transition between two phases, each one rich in one species, at high enough pressures. We should bear in mind that once monomers or 
dimers of small particles become located between two big squares, they will not be able to escape from the cage formed by big particles, due to the impossibility of small and big squares passing each other. Thus, if an equimolar mixture is initially prepared in a configuration where particles are randomly positioned (and consequently there is a high probability to find many small particles between the large ones) at high enough packing fraction, the mixture will become thermodynamically unstable with respect to phase segregation. However, the system will be unable to reach equilibrium (with two phases laterally segregated), as predicted from the thermodynamical analysis, due to severe particle jamming. These equilibrium states are not accessible in our strictly two-dimensional system. However, our system could still approximately describe an experimental realization consisting of a colloidal binary mixture of hard cubes sedimented in a container with a nanosculpted bottom surface under microgravity conditions. The experimental design of nanocolloids with arbitrary shape is a well-established procedure. In particular, an experiment on self-assembly of colloidal cubes via vertical deposition was reported in [49]. These or similar experimentally designed mixtures of colloidal cubes can be sedimented on the nanostructured surface with quasi2D channels such that monolayers of deposited cubes inside each channel are in contact with a "bath" of particles. The fact that particles can now enter or escape from the channel avoids the jamming effect and the mixture could reach a final state with two segregated "phases" inside the channel. Note that the system would not be strictly two dimensional as the channel would interact with the bulk regions in a nontrivial manner. Obviously, inside the channel cubes can rotate, so our model cannot strictly describe the real situation. However, the channel is so narrow and the packing fraction of confined cubes (for which interesting phenomena exist) so high that the orientational degrees of freedom are severely affected. Thus we hope that our predictions on the phase behavior and packing structures of the confined mixture are still valid. Although rotational degrees of freedom complicate the DF or TMM formalism, they can be included, in line with the works presented in Refs. [44,50,51].

Finally, a dynamical study of extremely confined parallel hard squares, in line with the work on hard-disk mixtures [52], could confirm the presence of locally jammed configurations of small particles inside cages formed by the big ones. These configurations would certainly induce an anomalous and highly anisotropic diffusion of particles inside the channel, a study which we leave for future work.

\section{ACKNOWLEDGMENTS}

Financial support from Ministerio de Economía, Industria y Competitividad (Spain) through Grants No. FIS201566523-P, No. PGC2018-096606-B-100, and No. FIS201786007-C3-1-P is acknowledged. We gratefully acknowledge fruitful discussions with P. Gurin and S. Varga.

\section{APPENDIX A: UNIFORM PHASES AT THE BULK PHASE}

For uniform densities $\rho_{i}$, the ideal and excess parts of the free-energy density, and the fluid pressure, are given by

$$
\begin{aligned}
\Phi_{\mathrm{id}} & =\rho\left(\ln \rho-1+\sum_{i} \mathbf{x}_{i} \ln \mathrm{x}_{i}\right), \\
\Phi_{\mathrm{exc}} & =-\ln (1-\eta)+\frac{n_{1}^{2}}{1-\eta}, \\
\beta p & =\frac{\rho}{1-\eta}+\frac{n_{1}^{2}}{(1-\eta)^{2}},
\end{aligned}
$$

where we have defined $n_{1}=n_{1 x}=n_{1 y}=\sum_{i} \rho_{i} \sigma_{i}$. Thus, the Gibbs free energy per particle for a fixed pressure $p_{0}$ in reduced thermal units can be calculated as

$$
\begin{aligned}
g \equiv & \frac{\beta G}{N}=\frac{\Phi_{\mathrm{id}}+\Phi_{\mathrm{exc}}+\beta p_{0}}{\rho} \\
= & \ln \rho^{*}-1+\sum_{i} \mathrm{x}_{i} \ln \mathrm{x}_{i}-\ln \left(1-\rho^{*} s_{2}\right) \\
& +\frac{\rho^{*} s_{1}^{2}}{1-\rho^{*} s_{2}}+\frac{p_{0}^{*}}{\rho^{*}},
\end{aligned}
$$

where we have defined the dimensionless number density and pressures as $\rho^{*}=\rho \sigma_{1}^{2}$ and $p_{0}^{*}=\beta p_{0} \sigma_{1}^{2}$. Also, we have defined the quantities

$$
s_{m} \equiv \sum_{i} \mathbf{x}_{i}\left(\frac{\sigma_{i}}{\sigma_{1}}\right)^{m}=\lambda^{m}-\left(\lambda^{m}-1\right) \mathbf{x}
$$

for $m=\{1,2\}$, with the aspect ratio of the mixture defined as $\lambda=\sigma_{2} / \sigma_{1}$. From the constant pressure condition we can calculate $\rho^{*}$ as a function of the composition, which results in

$$
\rho^{*}=\frac{1+2 p_{0}^{*} s_{2}-\sqrt{1+4 p_{0}^{*} s_{1}^{2}}}{2\left(p_{0}^{*} s_{2}^{2}+s_{2}-s_{1}^{2}\right)} .
$$

After substitution of (A4) into Eq. (A2) we obtain

$$
\begin{aligned}
g(\mathbf{x})= & p_{0}^{*} s_{2}+\sum_{i} \mathbf{x}_{i} \ln \mathbf{x}_{i}+\ln \left(\frac{\sqrt{1+4 p_{0}^{*} s_{1}^{2}}-1}{2 s_{1}^{2}}\right) \\
& +\sqrt{1+4 p_{0}^{*} s_{1}^{2}}-1 .
\end{aligned}
$$

It can be easily shown that the second derivative of $g(\mathbf{X})$ with respect to $\mathrm{X}$ gives the condition

$$
\begin{aligned}
\frac{d^{2} g}{d \mathbf{x}^{2}}(\mathbf{x})= & \frac{1}{\mathbf{x}(1-\mathbf{x})}+\left(\frac{\lambda-1}{s_{1}}\right)^{2} \\
& \times\left[1-\frac{1}{\sqrt{1+4 p_{0}^{*} s_{1}^{2}}}\right]>0 \forall\left\{\mathbf{x}, p_{0}^{*}, \lambda\right\} .
\end{aligned}
$$

Thus the Gibbs free energy per particle is always a convex function of composition and consequently we can draw the important conclusion that the PHS fluid is always stable with respect to phase separation.

\section{APPENDIX B: SPINODAL INSTABILITIES TO BULK NONUNIFORM PHASES}

We consider here the instability of the fluid phase with respect to inhomogeneities in one direction, say, $y$. We need 
to calculate the direct correlation functions

$$
\begin{aligned}
-c_{i j}\left(y-y^{\prime}\right) & =\frac{\delta^{2} \beta \mathcal{F}\left[\left\{\rho_{i}\right\}\right]}{\delta \rho_{i}(y) \delta \rho_{j}\left(y^{\prime}\right)} \\
& =\sum_{\alpha \beta} \Phi_{\alpha \beta}\left[\omega_{i}^{(\alpha)} * \omega_{j}^{(\beta)}\right]\left(y-y^{\prime}\right),
\end{aligned}
$$

where we have defined $\Phi_{\alpha \beta}=\frac{\partial^{2} \Phi_{\mathrm{exc}}}{\partial n_{\text {c }} \partial n_{\beta}}$ in the uniform limit and the symbol $*$ stands for convolution. The weighting functions $\omega_{i}^{(\alpha)}(y)$ are those which define the weighted densities through convolutions:

$$
n_{\alpha}(y)=\sum_{i}\left[\rho_{i} * \omega_{i}^{(\alpha)}\right](y) .
$$

They have the explicit forms

$$
\begin{aligned}
\omega_{i}^{(0)}(y) & =\frac{1}{2} \delta\left(\frac{\sigma_{i}}{2}-|y|\right), \\
\omega_{i}^{(2)}(y) & =\sigma_{i} \Theta\left(\frac{\sigma_{i}}{2}-|y|\right), \\
\omega_{i}^{(1 x)}(y) & =\frac{\sigma_{i}}{2} \delta\left(\frac{\sigma_{i}}{2}-|y|\right), \\
\omega_{i}^{(1 y)}(y) & =\Theta\left(\frac{\sigma_{i}}{2}-|y|\right),
\end{aligned}
$$

with $\delta(y)$ and $\Theta(y)$ the Dirac delta and Heaviside functions, respectively. The Fourier transforms of the functions $c_{i j}(y)$ give

$$
-\hat{c}_{i j}(q)=\sum_{\alpha, \beta} \Phi_{\alpha \beta} \hat{\omega}_{i}^{(\alpha)}(q) \hat{\omega}_{j}^{(\beta)}(q),
$$

where $q$ is the wave number, while

$$
\begin{aligned}
& \hat{\omega}_{i}^{(0)}(q)=\cos \left(\frac{q \sigma_{i}}{2}\right), \\
& \hat{\omega}_{i}^{(2)}(q)=\frac{2 \sigma_{i}}{q} \sin \left(\frac{q \sigma_{i}}{2}\right),
\end{aligned}
$$

$$
\begin{aligned}
& \hat{\omega}_{i}^{(1 x)}(q)=\sigma_{i} \cos \left(\frac{q \sigma_{i}}{2}\right), \\
& \hat{\omega}_{i}^{(1 y)}(q)=\frac{2}{q} \sin \left(\frac{q \sigma_{i}}{2}\right) .
\end{aligned}
$$

After a little algebra we arrive at

$$
\begin{aligned}
-\hat{c}_{i j}(q)= & \frac{2}{1-\eta}\left(\sigma_{i}+\sigma_{j}+\frac{n_{1}}{1-\eta} \sigma_{i} \sigma_{j}\right) \frac{\sin \left[q\left(\sigma_{i}+\sigma_{j}\right) / 2\right]}{q} \\
& +\frac{4}{(1-\eta)^{2}}\left[n_{1}\left(\sigma_{i}+\sigma_{j}\right)+\left(\rho+\frac{2 n_{1}^{2}}{1-\eta}\right) \sigma_{i} \sigma_{j}\right] \\
& \times \frac{\sin \left(q \sigma_{i} / 2\right) \sin \left(q \sigma_{j} / 2\right)}{q^{2}} .
\end{aligned}
$$

The determinant of the inverse structure factor matrix

$$
S_{i j}^{-1}(q, \eta)=\delta_{i j}-\sqrt{\rho_{i} \rho_{j}} \hat{c}_{i j}(q)
$$

can be calculated as

$$
\begin{aligned}
\mathcal{S}(q, \eta) \equiv & \operatorname{det}\left[S_{i j}^{-1}\right](q)=1-\rho_{1} \hat{c}_{11}(q)-\rho_{2} \hat{c}_{22}(q) \\
& +\rho_{1} \rho_{2}\left[\hat{c}_{11}(q) \hat{c}_{22}(q)-\hat{c}_{12}(q)^{2}\right] .
\end{aligned}
$$

Thus, the minimum value of $\eta$ for which the equations

$$
\mathcal{S}(q, \eta)=0, \quad \frac{\partial \mathcal{S}}{\partial q}(q, \eta)=0
$$

are fulfilled at the absolute minimum of $\mathcal{S}(q, \eta)$ as a function of $q$ provides the values $q^{*}$ and $\eta^{*}$ at bifurcation. These calculations are done by fixing the molar fraction of the mixture $\mathrm{x}$. Varying $\mathrm{X}$ and solving Eq. (B8), we find the spinodal curve $\eta^{*}(\mathbf{x})$ and the periodicity of the nonuniform phases $d(\mathbf{x}) \equiv$ $2 \pi / q^{*}(\mathbf{x})$. Note that if we fix the periodicity of the density profiles as $\rho_{i}(y+d)=\rho_{i}(y)$, as we have done at the end of Sec. III B, we need to solve only the first of Eqs. (B9).
[1] K. Zhao, R. Bruisma, and T. G. Mason, Nat. Commun. 3, 801 (2012).

[2] P. Y. Wang and T. G. Mason, J. Am. Chem. Soc. 137, 15308 (2015).

[3] L. Rossi and T. G. Mason, Soft Matter 11, 2461 (2015).

[4] W. Qi, J. de Graaf, F. Qiao, S. Marras, L. Manna, and M. Dijkstra, Nano Lett. 12, 5299 (2012).

[5] V. N. Manoharan, Science 349, 1253751 (2015).

[6] K. W. Wojciechowski and D. Frenkel, Comput. Methods Sci. Technol. 10, 235 (2004).

[7] Y. Martínez-Ratón, E. Velasco, and L. Mederos, J. Chem. Phys. 122, 014903 (2005).

[8] A. Donev, J. Burton, F. H. Stillinger, and S. Torquato, Phys. Rev. B 73, 054109 (2006).

[9] C. Avendaño and F. A. Escobedo, Soft Matter 8, 4675 (2012).

[10] A. P. Gantapara, W. Qi, and M. Dijkstra, Soft Matter 11, 8684 (2015).

[11] Y. Martínez-Ratón, A. Díaz-De Armas, and E. Velasco, Phys. Rev. E 97, 052703 (2018).

[12] J. A. Anderson, J. Antonaglia, J. A. Millan, M. Engel, and S. C. Glotzer, Phys. Rev. X 7, 021001 (2017).
[13] W. Shen, J. Antonaglia, J. A. Anderson, M. Engel, G. van Anders, and S. C. Glotzer, Soft Matter 15, 2571 (2019).

[14] V. Thapar, T. Hanrath, and F. A. Escobedo, Soft Matter 11, 1481 (2015).

[15] W. G. Hoover and A. G. de Rocco, J. Chem. Phys. 36, 3141 (1962).

[16] W. G. Hoover and J. C. Poirier, J. Chem. Phys. 38, 327 (1963).

[17] L. Lafuente and J. A. Cuesta, J. Chem. Phys. 119, 10832 (2003).

[18] K. Ramola and D. Dhar, Phys. Rev. E 86, 031135 (2012).

[19] S. Singh and S. R. Jain, J. Stat. Mech. (2018) 123102.

[20] W. G. Hoover, C. G. Hoover, and M. N. Bannerman, J. Stat. Phys. 136, 715 (2009).

[21] S. Belli, M. Dijkstra, and R. van Roij, J. Chem. Phys. 137, 124506 (2012).

[22] M. González-Pinto, Y. Martínez-Ratón, and E. Velasco, Soft Matter 13, 9246 (2017).

[23] K. Zhao, R. Bruinsma, and T. G. Mason, Proc. Natl. Acad. Sci. USA 108, 2684 (2011).

[24] A. Buhot and W. Krauth, Phys. Rev. E 59, 2939 (1999).

[25] D. de las Heras, L. Mederos, and E. Velasco, Liq. Cryst. 37, 45 (2009). 
[26] D. de las Heras, E. Velasco, and L. Mederos, Phys. Rev. E 79, 061703 (2009).

[27] T. Geigenfeind, S. Rosenzweig, M. Schmidt, and D. de las Heras, J. Chem. Phys. 142, 174701 (2015).

[28] I. C. Garlea and B. M. Mulder, Soft Matter 11, 608 (2015).

[29] A. H. Lewis, I. Garlea, J. Alvarado, O. J. Oliver, P. D. Howell, A. Majumdar, B. M. Mulder, M. P. Lettinga, G. H. Koenderink, and D. G. A. L. Aarts, Soft Matter 10, 7865 (2014).

[30] O. V. Manyuhina, K. B. Lawlor, M. C. Marchetti, and B. J. Bowick, Soft Matter 11, 6099 (2015).

[31] D. de las Heras and E. Velasco, Soft Matter 10, 1758 (2014).

[32] M. González-Pinto, Y. Martínez-Ratón, and E. Velasco, Phys. Rev. E 88, 032506 (2013).

[33] D. A. Kofke and A. J. Post, J. Chem. Phys. 98, 4853 (1993).

[34] P. Gurin and S. Varga, J. Chem. Phys. 139, 244708 (2013).

[35] P. Gurin and S. Varga, J. Chem. Phys. 142, 224503 (2015).

[36] M. J. Godfrey and M. A. Moore, Phys. Rev. E 91, 022120 (2015).

[37] P. Gurin, S. Varga, and G. Odriozola, Phys. Rev. E 94, 050603(R) (2016).

[38] P. Gurin, G. Odriozola, and S. Varga, Phys. Rev. E 95, 042610 (2017).

[39] P. Gurin, S. Varga, M. González-Pinto, Y. Martínez-Ratón, and E. Velasco, J. Chem. Phys. 146, 134503 (2017).

[40] Y. Hu, L. Fu, and P. Charbonneau, Mol. Phys. 116, 3345 (2018).
[41] R. Roth, K. Mecke, and M. Oettel, J. Chem. Phys. 136, 081101 (2012).

[42] J. A. Cuesta and Y. Martínez-Ratón, J. Chem. Phys. 107, 6379 (1997).

[43] Y. Martínez-Ratón, Phys. Rev. E 69, 061712 (2004).

[44] R. Wittmann, C. E. Sitta, F. Smallenburg, and H. Löwen, J. Chem. Phys. 147, 134908 (2017).

[45] R. Roth, J. Phys.: Condens. Matter 22, 063102 (2010).

[46] P. Tarazona, J. A. Cuesta, and Y. Martínez-Ratón, in Theory and Simulation of Hard-Sphere Fluids and Related Systems, edited by Á. Mulero, Lecture Notes in Physics Vol. 753 (Springer, Berlin, 2008), pp. 247-341.

[47] M. González-Pinto, Y. Martínez-Ratón, S. Varga, P. Gurin, and E. Velasco, J. Phys.: Condens. Matter 28, 244002 (2016).

[48] P. Gurin, S. Varga, Y. Martínez-Ratón, and E. Velasco, Phys. Rev. E 97, 052606 (2018).

[49] J.-M. Meijer, F. Hagemans, L. Rossi, D. V. Byelov, S. I. R. Castillo, A. Snigirev. I. Snigireva, A. P. Philipse, and A. V. Petukhov, Langmuir 28, 7631 (2012).

[50] L. M. Casey and L. K. Runnels, J. Chem. Phys. 51, 5070 (1969).

[51] J. L. Lebowitz, J. K. Percus, and J. Talbot, J. Stat. Phys. 49, 1221 (1987).

[52] C. D. Ball, N. D. MacWilliam, J. K. Percus, and R. K. Bowles, J. Chem. Phys. 130, 054504 (2009). 